Article

\title{
From Quinoxaline, Pyrido[2,3-b]pyrazine and Pyrido[3,4-b]pyrazine to Pyrazino-Fused Carbazoles and Carbolines
}

\author{
Frédéric Lassagne ${ }^{1, *}$, Timothy Langlais ${ }^{1}$, Elsa Caytan ${ }^{1}{ }^{[}$, Emmanuelle Limanton ${ }^{1}$, \\ Ludovic Paquin ${ }^{1, *}$, Manon Boullard ${ }^{1} \oplus$, Coline Courtel ${ }^{1}$, Idriss Curbet ${ }^{1}$, Clément Gédéon ${ }^{1}$, \\ Julien Lebreton ${ }^{1}$, Laurent Picot ${ }^{2, *}$, Valérie Thiéry ${ }^{2}$ (D), Mohamed Souab ${ }^{3}$, Blandine Baratte ${ }^{3}$, \\ Sandrine Ruchaud ${ }^{3}$, Stéphane Bach ${ }^{3, *(D)}$, Thierry Roisnel ${ }^{1}$ and Florence Mongin $1, *(\mathbb{D})$
}

1 Univ Rennes, CNRS, Institut des Sciences Chimiques de Rennes (ISCR)-UMR 6226, F-35000 Rennes, France; timothy.langlais@etudiant.univ-rennes1.fr (T.L.); elsa.caytan@univ-rennes1.fr (E.C.); emmanuelle.limanton@univ-rennes1.fr (E.L.); manon.boullard@etudiant.univ-rennes1.fr (M.B.); coline.courtel@etudiant.univ-rennes1.fr (C.C.); idriss.curbet@etudiant.univ-rennes1.fr (I.C.); clement.gedeon@etudiant.univ-rennes1.fr (C.G.); julien.lebreton@etudiant.univ-rennes1.fr (J.L.); thierry.roisnel@univ-rennes1.fr (T.R.)

2 Laboratoire Littoral Environnement et Sociétés, UMRi CNRS 7266, Université de La Rochelle, 17042 La Rochelle, France; valerie.thiery@univ-lr.fr

3 Sorbonne Universités, UPMC Univ Paris 06, CNRS USR3151, "Protein Phosphorylation and Human Disease" Unit, Plateforme de criblage KISSf, Station Biologique de Roscoff, Place Georges Teissier, 29688 Roscoff, France; mo.souab@gmail.com (M.S.); baratte@sb-roscoff.fr (B.B.); sandrine.ruchaud@sb-roscoff.fr (S.R.)

* Correspondence: frederic.lassagne@univ-rennes1.fr (F.L.); ludovic.paquin@univ-rennes.fr (L.P.); laurent.picot@univ-lr.fr (L.P.); bach@sb-roscoff.fr (S.B.); florence.mongin@univ-rennes1.fr (F.M.); Tel.: +33-223-236-931 (F.M.)

Academic Editor: Richard A. Bunce

Received: 23 October 2018; Accepted: 11 November 2018; Published: 13 November 2018

\begin{abstract}
Diphenylated quinoxaline, pyrido[2,3-b]pyrazine and 8-bromopyrido[3,4-b]pyrazine were halogenated in deprotometalation-trapping reactions using mixed 2,2,6,6-tetramethyl piperidino-based lithium-zinc combinations in tetrahydrofuran. The 2,3-diphenylated 5-iodoquinoxaline, 8-iodopyrido[2,3-b]pyrazine and 8-bromo-7-iodopyrido[3,4-b]pyrazine thus obtained were subjected to palladium-catalyzed couplings with arylboronic acids or anilines, and possible subsequent cyclizations to afford the corresponding pyrazino[2,3-a]carbazole, pyrazino $\left[2^{\prime}, 3^{\prime}: 5,6\right]$ pyrido[4,3- $b$ ]indole and pyrazino $\left[2^{\prime}, 3^{\prime}: 4,5\right]$ pyrido[2,3- $\left.d\right]$ indole, respectively. 8-Iodopyrido[2,3- $\left.b\right]$ pyrazine was subjected either to a copper-catalyzed C-N bond formation with azoles, or to direct substitution to introduce alkylamino, benzylamino, hydrazine and aryloxy groups at the 8 position. The 8-hydrazino product was converted into aryl hydrazones. Most of the compounds were evaluated for their biological properties (antiproliferative activity in A2058 melanoma cells and disease-relevant kinase inhibition).
\end{abstract}

Keywords: pyrazine; deprotometalation; coupling; $N$-arylation; palladium; copper

\section{Introduction}

Quinoxalines and pyridopyrazines are aromatic heterocycles present in compounds endowed with numerous interesting properties. Some derivatives are bioactive and are used as antimicrobial, anti-inflammatory, antimalarial, anticancer and antidepressant compounds [1,2]. Others are for example employed as organic dyes [3], electroluminescent materials [4], and organic semiconductors [5]. Quinoxaline and pyridopyrazine substrates can be readily synthesized by 
condensation of 1,2-dicarbonyl compounds with 1,2-arylenediamines [6] and lend themselves to further elaboration.

Deprotonative lithiation followed by interception of the arylmetals with electrophiles is an efficient way to functionalize aromatic compounds [7-12]. However, reactions with substrates sensitive to nucleophilic attack such as azines must be performed at very low temperatures to avoid secondary reactions between arylmetals and functions [13-15]. The use of in situ metal traps avoids the use of cryogenic conditions to achieve these reactions $[16,17]$. We have developed mixed lithium-zinc combinations based on TMP (TMP = 2,2,6,6-tetramethylpiperidino) capable of deprotonating sensitive substrates at temperatures close to rt [18-21]. In order to obtain original scaffolds such as pyrazino-fused carbazoles and carbolines, we decided to combine this deprotometalation under in situ trapping conditions with palladium- and copper-catalyzed coupling reactions.

\section{Results and Discussion}

\subsection{Synthesis}

To functionalize 2,3-diphenylquinoxaline (1a) and 2,3-diphenylpyrido[2,3-b]pyrazine (2a), two deprotonation methods were tested in tetrahydrofuran (THF) (Table 1, Method A and Method B).

Table 1. Deprotonative metalation of 2,3-diphenylquinoxaline (1a) and 2,3-diphenylpyrido[2,3-b] -pyrazine (2a) and conversion to the halogeno derivatives.

\section{Method A}<smiles>[X]c1cccc2nc(-c3ccccc3)c(-c3ccccc3)nc12</smiles>

1a: $\mathrm{X}=\mathrm{CH}$

2a: $X=N$
1) base prepared from $\mathrm{ZnCl}_{2} \cdot$ TMEDA ( 0.5 equiv) and LiTMP (1.5 equiv), THF, rt, $2 \mathrm{~h}$

2) Electrophile, conditions

\section{Method B}

1) $\mathrm{ZnCl}_{2} \cdot$ TMEDA (1 equiv)

2) LiTMP (1.2 equiv), THF, $-20^{\circ} \mathrm{C}, 30 \mathrm{~min}$

3) Electrophile, conditions<smiles>Fc1cccc2nc(-c3ccccc3)c(-c3ccccc3)nc12</smiles>

1b: $\mathrm{X}=\mathrm{CH}$

2b: $X=N$

\begin{tabular}{|c|c|c|c|c|}
\hline Entry & Substrate & Method & Electrophile, Conditions & Product (E), Yield $(\%)^{1}$ \\
\hline 1 & $\mathbf{1 a}(\mathrm{X}=\mathrm{CH})$ & A & $\mathrm{I}_{2}, \mathrm{THF}, 0{ }^{\circ} \mathrm{C}, 1 \mathrm{~h}$ & $\mathbf{1 b}(\mathrm{I}), 74^{2}$ \\
\hline 2 & $\mathbf{1 a}(\mathrm{X}=\mathrm{CH})$ & B & $\mathrm{I}_{2}, \mathrm{THF}, 0^{\circ} \mathrm{C}, 1 \mathrm{~h}$ & $1 \mathbf{b}(\mathrm{I}), 70$ \\
\hline 3 & $2 \mathbf{a}(X=N)$ & A & $\mathrm{I}_{2}, \mathrm{THF}, 0^{\circ} \mathrm{C}, 1 \mathrm{~h}$ & 2b-I (I), 70 \\
\hline 4 & $2 \mathbf{a}(X=N)$ & B & $\mathrm{I}_{2}, \mathrm{THF}, 0^{\circ} \mathrm{C}, 1 \mathrm{~h}$ & 2b-I (I), 62 \\
\hline 5 & $2 \mathbf{a}(X=N)$ & B & $\mathrm{Br}_{2},-20^{\circ} \mathrm{C}, 1 \mathrm{~h}$ & $\begin{array}{c}2 b-\mathrm{Br}(\mathrm{Br}), \\
60\end{array}$ \\
\hline 6 & $2 \mathbf{a}(X=N)$ & B & $-\mathrm{Cl}-20^{\circ} \mathrm{C}, 1 \mathrm{~h}$ & $\begin{array}{c}2 \mathrm{~b}-\mathrm{Cl}(\mathrm{Cl}), \\
62\end{array}$ \\
\hline
\end{tabular}

${ }^{1}$ After purification (see experimental part). ${ }^{2}$ The rest is 5,8-diiodo-2,3-diphenylquinoxaline (1 $\mathbf{b}^{\prime} ; \mathbf{7} \%$ yield; see Figure 1). $\mathbf{1 b}^{\prime}$ was isolated in $70 \%$ yield by using $\mathrm{ZnCl}_{2}$.TMEDA (1 equiv) and LiTMP (3 equiv).

The lithium-zinc base of Method $A$ is prepared from $\mathrm{ZnCl}_{2} \cdot$ TMEDA (TMEDA $=N, N, N^{\prime}, N^{\prime}-$ tetramethylethylenediamine) and LiTMP in a 1:3 ratio. Previous studies have suggested that it 
is a 1:1 LiTMP-Zn(TMP) $)_{2}$ combination. While LiTMP deprotonates the substrate, $\mathrm{Zn}(\mathrm{TMP})_{2}$ intercepts the generated aryllithium $[18,19,22]$. A recent computer study on anisole showed that the reactive species is solvated LiTMP. The effectiveness of the reaction derives from the stabilizing effect of the transmetalation step [21].

It is possible to replace $\mathrm{Zn}(\mathrm{TMP})_{2}$ by $\mathrm{ZnCl}_{2}$ provided that there is no contact between LiTMP and $\mathrm{ZnCl}_{2}$ in the absence of the aromatic compound [23,24]. Thus, Method B is limited to activated substrates for which deprotonation is favored over reaction between LiTMP and $\mathrm{ZnCl}_{2}$.

Whereas Method $A$ should provide a lithium arylzincate, Method B should rather generate an arylzinc. Nevertheless, both species are known to react with iodine by aryl transfer.

Thus, 2,3-diphenylquinoxaline (1a) and 2,3-diphenylpyrido[2,3-b]pyrazine (2a) were involved in Method $A$. After treatment at $\mathrm{rt}$ with the base for $2 \mathrm{~h}$, addition of iodine led to iodoquinoxaline $\mathbf{1 b}$ and iodopyrido[2,3-b] pyrazine $2 \mathrm{~b}-\mathrm{I}$ in 74 and 70\% yield, respectively (entries 1 and 3 ).

To evaluate Method $B, \mathbf{1 a}$ and $\mathbf{2 a}$ were mixed with $\mathrm{ZnCl}_{2} \cdot$ TMEDA before addition of LiTMP at $-20{ }^{\circ} \mathrm{C}$ and stirring for $0.5 \mathrm{~h}$ (Method B, entries 2 and 4). After subsequent interception with iodine, $\mathbf{1 b}$ and $\mathbf{2 b - I}$ were isolated in 70 and 62\% yield, respectively (entries 2 and 4).

We explored the use of other electrophiles to intercept the heteroarylzinc chloride prepared from $\mathbf{2 a}$ by using Method B. Conversion to the corresponding bromide $\mathbf{2 b - B r}$ (60\% yield, entry 5) and chloride 2b-Cl (62\% yield, entry 6) was performed using bromine and trichloroisocyanuric acid, respectively, as the electrophile.

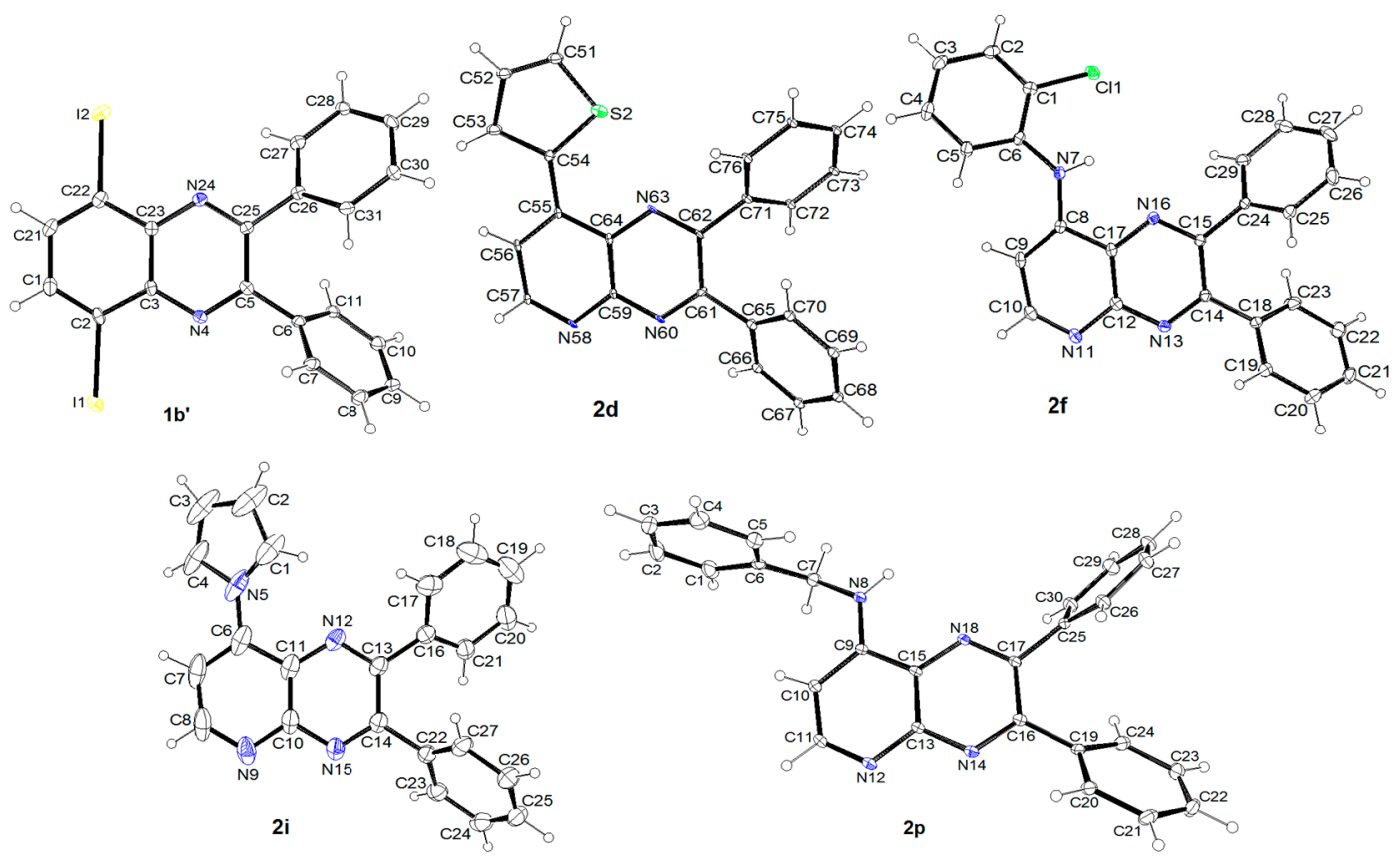

Figure 1. ORTEP diagrams (30\% probability) of $\mathbf{1} \mathbf{b}^{\prime}, \mathbf{2 d}, \mathbf{2} \mathbf{f}, \mathbf{2} \mathbf{i}, \mathbf{2} \mathbf{p}$.

The deprotometalation-iodination sequence was successfully applied to 8-bromo-2,3-diphenyl pyrido[3,4-b]pyrazine (3a) [25,26], but failed with 7-bromo-2,3-diphenylpyrido[2,3- $b$ ]pyrazine (4a) due to significant degradation before trapping (Scheme 1 ). While the position of the iodo group in $\mathbf{3 b}$ was evidenced by subsequent reaction, it was studied by advanced NMR experiments in the case of $4 \mathbf{b}$ (see Supplementary Materials).

In order to prepare original pyrazino-fused carbazoles and carbolines, iodides $\mathbf{1 b}$ and $\mathbf{2} \mathbf{b}$-I were subjected to in Suzuki couplings [27,28] under standard conditions (Table 2) [29]. Phenyl- (entry 1), 2-thienyl- (entries 2 and 3) and 2-aminophenyl- (entries 4 and 5) boronic acids led to the 5-arylated derivatives 1c-e and $\mathbf{2 d}$,e in $42-97 \%$ yields. The more electron-rich arylboronic acids and the less electron-poor quinoxaline substrate $\mathbf{1 b}$ gave the best results. 
<smiles>Brc1cncc2nc(-c3ccccc3)c(-c3ccccc3)nc12</smiles>

$3 a$

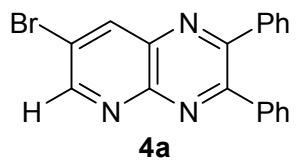

(67\% yield)

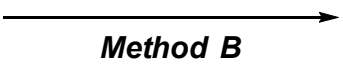

1) $\mathrm{ZnCl}_{2} \cdot \mathrm{TMEDA}(1$ equiv)

2) LiTMP (1.2 equiv)

THF, $-20^{\circ} \mathrm{C}, 30 \mathrm{~min}$

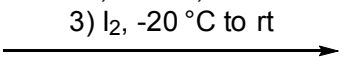

$(5 \%$ yield)<smiles>Brc1c(I)ncc2nc(-c3ccccc3)c(-c3ccccc3)nc12</smiles>

3b<smiles>Brc1cc2nc(-c3ccccc3)c(-c3ccccc3)nc2nc1I</smiles>

Scheme 1. Deprotonative metalation of 8-bromo-2,3-diphenylpyrido[3,4-b]pyrazine (3a) and 7-bromo-2,3-diphenylpyrido[2,3-b]pyrazine (4a) followed by conversion to the halogeno derivatives.

Table 2. Suzuki coupling from 5-iodo-2,3-diphenylquinoxaline (1b) and 8-iodo-2,3-diphenyl pyrido[2,3-b]pyrazine (2b-I).

\begin{tabular}{|c|c|c|c|}
\hline & $\begin{array}{l}\text { 1b: } X=C H \\
\text { 2b-l: } X=N\end{array}$ & $\begin{array}{c}\mathrm{ArB}(\mathrm{OH})_{2} \\
\mathrm{Pd}\left(\mathrm{PPh}_{3}\right)_{4}(5 \text { mol\% }) \\
\mathrm{NaHCO}_{3}(4 \text { equiv }) \\
\mathrm{DME}-\mathrm{H}_{2} \mathrm{O} \\
80^{\circ} \mathrm{C}, 3 \mathrm{~h}\end{array}$ & $\begin{array}{l}1 \mathrm{c}-\mathrm{e}: \mathrm{X}=\mathrm{CH} \\
2 \mathrm{~d}, \mathrm{e}: \mathrm{X}=\mathrm{N}\end{array}$ \\
\hline Entry & Substrate & $\operatorname{ArB}(\mathrm{OH})_{2}$ & Product (Ar), Yield (\%) ${ }^{1}$ \\
\hline 1 & $\mathbf{1 b}(\mathrm{X}=\mathrm{CH})$ & $\mathrm{PhB}(\mathrm{OH})_{2}$ & 1c (Ph), 42 \\
\hline 2 & $\mathbf{1 b}(\mathrm{X}=\mathrm{CH})$ & & 1d (2-thienyl), 97 \\
\hline 3 & $2 b-I(X=N)$ & $\mathrm{B}(\mathrm{OH})_{2}$ & 2d (2-thienyl) ${ }^{2}, 75$ \\
\hline 4 & $\mathbf{1 b}(\mathrm{X}=\mathrm{CH})$ & & 1e (2-aminophenyl), 92 \\
\hline 5 & 2b-I $(X=N)$ & $\mathrm{B}(\mathrm{OH})_{2}$ & 2e (2-aminophenyl), 73 \\
\hline
\end{tabular}

${ }^{1}$ After purification (see experimental part). ${ }^{2}$ See Figure 1.

No intramolecular nitrene insertion into the corresponding diazino-fused carbazole and $\beta$-carboline was obtained for the azides coming from 1e and $\mathbf{2 e}$ [29]. We thus turned to the synthesis of the original pyrazino[2,3- $a$ ]carbazole $\mathbf{1 g}$ and the corresponding pyrazino-fused $\gamma$-carboline $\mathbf{2 g}$ isomers by combining intermolecular C-N bond formation [30-38] with intramolecular C-C bond formation (Scheme 2).

The first step, attempted from $\mathbf{1 b}$ by using catalytic palladium(II) acetate as transition metal source, Xantphos as ligand, and sodium tert-butoxide as base in toluene [39], yielded only 16\% of diarylamine 1f. Applying to $\mathbf{1 b}$ and $\mathbf{2 b}$-I the conditions reported by Maes and co-workers for related reactions [29], If and $2 f$ were obtained in 92 and 67\% yield, respectively (Scheme 2, left). Inspired by Pieters and co-workers, who cyclized 4-(2-chlorophenylamino)pyridine into $5 H$-pyrido[4,3-b]indole under these conditions [40], we successfully employed catalytic $\left(\mathrm{Pd}_{2}(\mathrm{dba})_{3}\right)$ and tri-tert-butylphosphine as catalyst precursors, diazabicyclo[5.4.0]undec-7-ene (DBU) as base, and dioxane as solvent for the second step. After $10 \mathrm{~min}$ at $180{ }^{\circ} \mathrm{C}$ under microwave irradiation, the pyrazino-fused carbazole $\mathbf{1 g}$ and $\gamma$-carboline $\mathbf{2 g}$ were isolated in moderate yields (Scheme 2, right).

We decided to combine both steps in an auto-tandem process under microwave irradiation (Table 3$)$. Using $\left(\mathrm{Pd}_{2}(\mathrm{dba})_{3}\right)$, we selected Xantphos for its higher efficiency in comparison with tri-tert-butylphosphine. From $\mathbf{2 b}$, best results were obtained with three equivalents of DBU as base (entries 1 and 2). In addition, a longer reaction time was required to ensure complete conversion and this afforded carboline $\mathbf{2 g}$ in $70 \%$ yield (entry 3 ). 
<smiles>[X]c1ccc(I)c2nc(-c3ccccc3)c(-c3ccccc3)nc12</smiles>

1b: $\mathrm{X}=\mathrm{CH}$

2b-l: $X=N$

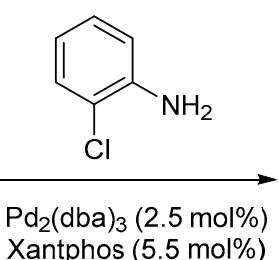

$\mathrm{Cs}_{2} \mathrm{CO}_{3}$ (3 equiv) dioxane, $110^{\circ} \mathrm{C}, 24 \mathrm{~h}$<smiles>[X]c1nc2c([X])ccc(Nc3ccccc3Cl)c2nc1-c1ccccc1</smiles>

$1 f(X=\mathrm{CH}): 92 \%$

$2 \mathbf{f}(\mathrm{X}=\mathrm{N}): 67 \%$

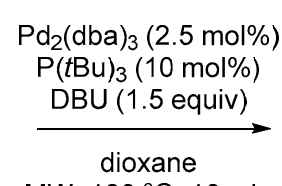

$\mathrm{MW}, 180^{\circ} \mathrm{C}, 10 \mathrm{~min}$

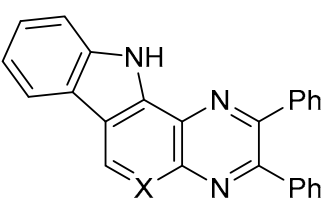

$1 \mathrm{~g}(\mathrm{X}=\mathrm{CH}): 62 \%$

$2 g(X=N): 65 \%$

Scheme 2. Conversion of 5-iodo-2,3-diphenylquinoxaline (1b) and 8-iodo-2,3-diphenylpyrido[2,3-b] pyrazine (2b-I) into 2,3-diphenyl-11H-pyrazino[2,3-a]carbazole (1g) and 2,3-diphenyl-11H-pyrazino $\left[2^{\prime}, 3^{\prime}: 5,6\right]$ pyrido[ $[4,3-b]$ indole $(2 \mathrm{~g})$, respectively. Dba $=$ dibenzylideneacetone.

Table 3. Study of the conversion of the 8-halogenated 2,3-diphenylpyrido[2,3-b]pyrazines $2 \mathbf{b}$ into 2,3-diphenyl-11H-pyrazino[2' $\left.3^{\prime}: 5,6\right]$ pyrido[4,3-b]indole (2g) under MW irradiation.

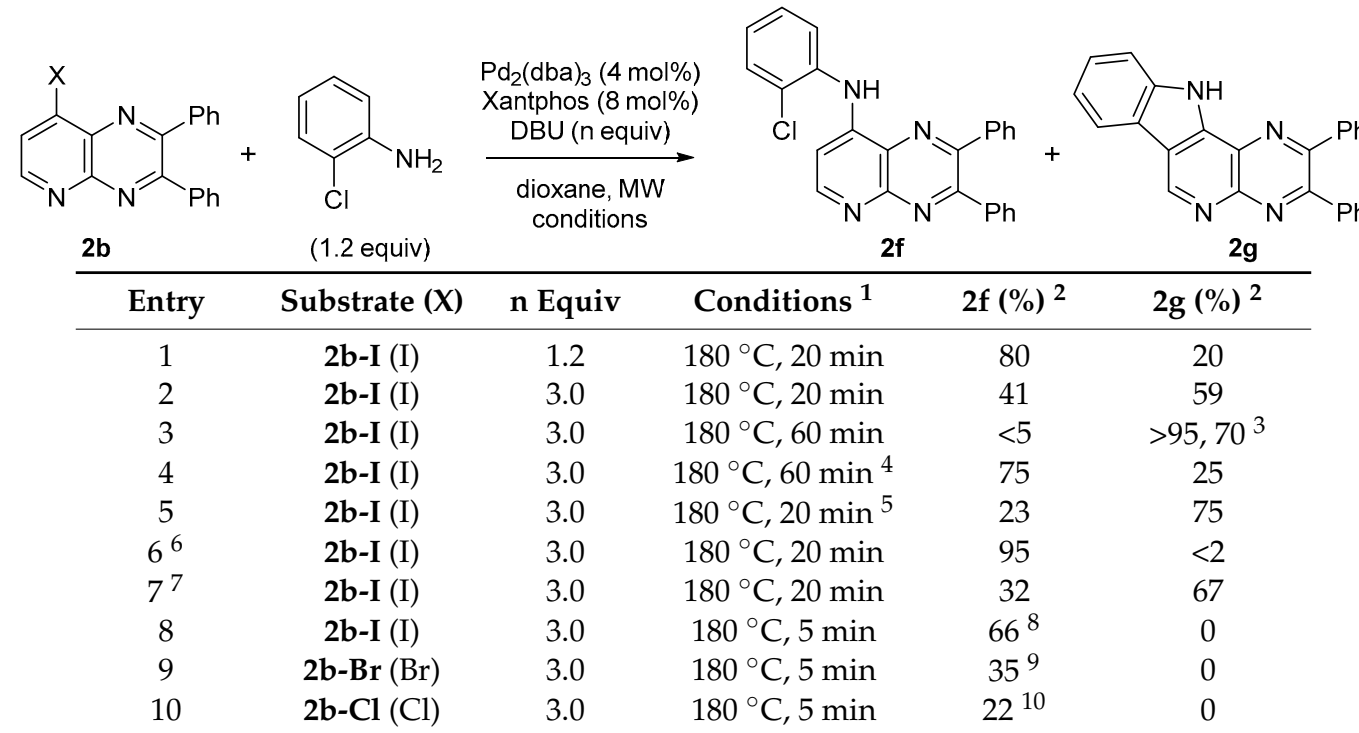

\footnotetext{
${ }^{1}$ Maximum microwave power applied: $150-200 \mathrm{~W}$ at the beginning to reach the required temperature. ${ }^{2}$ Evaluated from the NMR spectra of the crudes. ${ }^{3}$ Yield after purification. ${ }^{4}$ Microwave profile of irradiation: The sequence 'Maximum microwave power applied: $150-200 \mathrm{~W}$ to reach $180^{\circ} \mathrm{C}$ then 2 min at $180^{\circ} \mathrm{C}$ before cooling to $100^{\circ} \mathrm{C}^{\prime}$ was repeated every $4 \mathrm{~min} .{ }^{5}$ Then classical heating at $180{ }^{\circ} \mathrm{C}$ for $40 \mathrm{~min} .{ }^{6}$ Without catalyst. ${ }^{7}$ By using $12 \mathrm{~mol} \% \mathrm{Pd}_{2}(\mathrm{dba})_{3}$ and 30 mol\% Xantphos. ${ }^{8}$ The rest was unreacted $\mathbf{2 b}-\mathbf{I}(32 \%) .{ }^{9}$ The rest was unreacted $\mathbf{2 b}-\mathbf{B r}(38 \%)$ and $\mathbf{2 a}(28 \%) .{ }^{10}$ The rest was unreacted $\mathbf{2} \mathbf{b}-\mathbf{C l}(78 \%)$.
}

By testing a profile to maximize the microwave power, we noticed that an increase of the applied power favored the formation of $2 \mathbf{f}$ over $2 \mathbf{g}$ (entry 4 ). By carrying out one third of the reaction time under microwave irradiation and the rest by classical heating at the same temperature, a small microwave effect was evidenced (entry 5). While $\mathbf{2} \mathbf{g}$ was not formed without catalyst, $\mathrm{C}-\mathrm{N}$ bond formation giving $2 \mathbf{f}$ could take place (entry 6; see Figure 1). However, increasing the catalyst amount had no impact on the conversion to $\mathbf{2 g}$ (entry 7). Finally, we intentionally chose a short reaction time (5 $\mathrm{min}$ ) in order to compare the palladium-catalyzed reactions under microwave irradiation from $\mathbf{2 b}-\mathbf{I}$ (entry 7), $\mathbf{2 b}-\mathbf{B r}$ (entry 7 ) and $\mathbf{2 b}-\mathbf{C l}$ (entry 10 ). The results clearly showed decreasing reactivity from $\mathbf{2 b}-\mathbf{I}$ to $\mathbf{2} \mathbf{b}-\mathbf{C l}$, and thus, we selected iodo as halogeno group to pursue our investigations.

We applied the optimized procedure to the synthesis of the pyrazino-fused $\alpha$-carboline $\mathbf{3 g}$ from the bromoiodo substrate $\mathbf{3 b}$ and aniline. No trace of the expected product $3 \mathbf{g}$ was detected but the formation of $\mathbf{3} \mathbf{g}^{\prime}$ due to competitive debromination was noted, showing a less obvious intramolecular C-H arylation (Scheme 3, left). Consequently, we moved to the synthesis of the pyrazino-fused $\delta$-carboline $\mathbf{3 h}$. Upon treatment of $\mathbf{3 b}$ by 2 -aminophenylboronic acid under standard conditions [29], coupling and subsequent cyclization occurred, providing $3 \mathrm{~h}$ in $65 \%$ yield (Scheme 3 , right). 


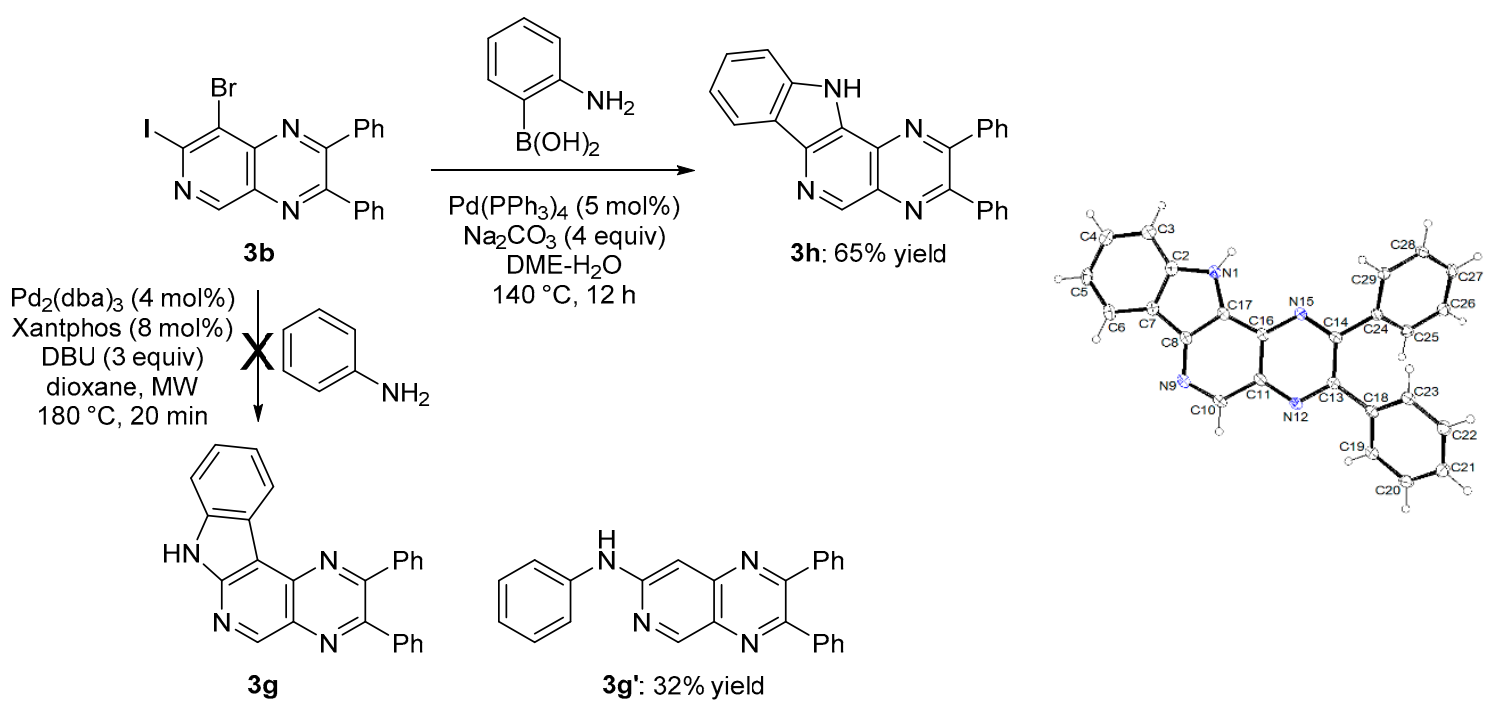

Scheme 3. Conversion of 8-bromo-7-iodo-2,3-diphenylpyrido[3,4- $b$ ]pyrazine (3b) into 2,3-diphenyl$11 H$-pyrazino $\left[2^{\prime}, 3^{\prime}: 4,5\right]$ pyrido[2,3-d]indole (3h) and ORTEP diagram (30\% probability) of $3 \mathbf{h}$.

To take advantage of the iodo group on $\mathbf{2 b - I}, \mathrm{C}-\mathrm{N}$ bond formation with azoles was attempted under copper catalysis as reported previously [41,42] (Table 4). Thus, by treating $\mathbf{2 b - I}$ with pyrrole (entry 1; see Figure 1), indole (entry 2), pyrazole (entry 3), imidazole (entry 4) or 1,2,4-triazole (entry 5 ), in the presence of catalytic copper(I) oxide, cesium carbonate, and dimethylsulfoxide (DMSO) at $110{ }^{\circ} \mathrm{C}$ for $24 \mathrm{~h}$, the expected $\mathrm{N}$-arylated azoles were obtained in 51 to $79 \%$ yields.

As previously mentioned [22], such reactions work far less efficiently when performed on diiodides. Indeed, reacting the diiodide $\mathbf{1} \mathbf{b}^{\prime}$ with pyrazole only gave the monofunctionalized derivative $\mathbf{1} \mathbf{k}^{\prime}$, regardless of the amount of azole employed (Scheme 4).<smiles>Ic1ccc(I)c2nc(-c3ccccc3)c(-c3ccccc3)nc12</smiles>

$1 b^{\prime}$

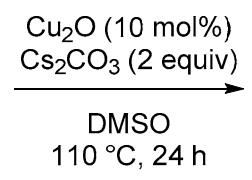

(50\% yield)<smiles>Ic1ccc(-n2cccn2)c2nc(-c3ccccc3)c(-c3ccccc3)nc12</smiles>

$1 k^{\prime}$

Scheme 4. Copper-catalyzed $N$-arylation of 5,8-diiodo-2,3-diphenylquinoxaline $\left(\mathbf{1 b}^{\prime}\right)$.

Different amines and hydrazine reacted with $\mathbf{2 b - I}$ without recourse to catalyst (Table 5), affording the corresponding secondary amines $\mathbf{2 n - p}$ (entries 1-3) and arylhydrazine $\mathbf{2 q}$ (entry 4) in good yields. The latter was converted into the hydrazones $\mathbf{2 r}-\mathbf{u}$ in the presence of aromatic aldehydes chosen for their ability to potentially interact with binding sites of biological interest [43] (Scheme 5). Finally, reaction of $\mathbf{2 b}$-I with a phenol also proved possible without catalyst, giving the diaryl ether $2 \mathbf{v}$ in $64 \%$ yield (Scheme 6). 
Table 4. Copper-catalyzed N-arylation of 8-iodo-2,3-diphenylpyrido[2,3-b]pyrazine (2b-I) using azoles.<smiles>Ic1ccnc2nc(-c3ccccc3)c(-c3ccccc3)nc12</smiles>

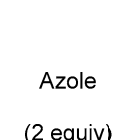

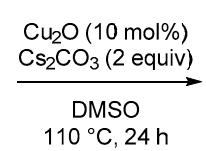<smiles>CCNc1ccnc2nc(-c3ccccc3)c(N(C)c3ccccc3)nc12</smiles>

Entry Azole

${ }^{1}$ After purification (see experimental part). The rest is starting material and the corresponding deiodinated compound.<smiles>NNc1ccnc2nc(-c3ccccc3)c(-c3ccccc3)nc12</smiles>

$2 q$<smiles>[R]c1ccccc1C=O</smiles>

(1.2 equiv)

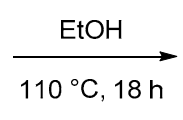<smiles>[R]c1cccc(/C=N/Nc2ccnc3nc(-c4ccccc4)c(-c4ccccc4)nc23)c1</smiles>

2r-u<smiles>CN=Cc1ccccc1O</smiles><smiles>N=Cc1ccc2c(c1)OCO2</smiles><smiles>NNc1ccnc2nc(-c3ccccc3)c(-c3ccccc3)nc12</smiles><smiles>N[AlH]</smiles><smiles>Cc1ccnc2nc(-c3ccccc3)c(-c3ccccc3)nc12</smiles>

2s, $70 \%$ yield
$\mathrm{MeO}$<smiles>CN=Cc1ccc(C)cc1O</smiles><smiles>NNc1ccnc2nc(-c3ccccc3)c(-c3ccccc3)nc12</smiles>

2t, $80 \%$ yield<smiles>FC(F)(F)c1ccc(/C=N/Nc2ccnc3nc(-c4ccccc4)c(-c4ccccc4)nc23)cc1</smiles>

$2 \mathrm{u}, 73 \%$ yield

Scheme 5. Conversion of 8-hydrazino-2,3-diphenylpyrido[2,3-b]pyrazine (2q) into aryl hydrazones. 
Table 5. Conversion of 8-iodo-2,3-diphenylpyrido[2,3-b]pyrazine (2b-I) into corresponding amines and hydrazine.

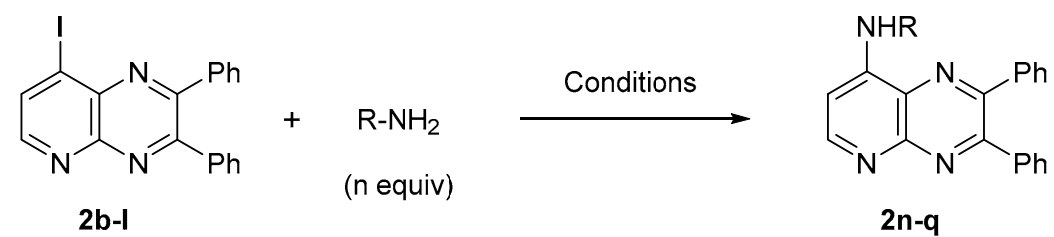

Entry Conditions

${ }^{1}$ After purification (see the Materials and Methods section). ${ }^{2}$ See Figure 1.<smiles>Ic1ccnc2nc(-c3ccccc3)c(-c3ccccc3)nc12</smiles>

2b-I<smiles>COc1ccc(O)c(C(C)=O)c1</smiles>

(1.1 equiv)<smiles>COc1ccc(Oc2ccnc3nc(-c4ccccc4)c(-c4ccccc4)nc23)c(C(C)=O)c1</smiles>

2v, $64 \%$ yield

Scheme 6. Conversion of 8-iodo-2,3-diphenylpyrido[2,3-b]pyrazine (2b-I) into ether $2 \mathbf{v}$.

\subsection{Biological Activity}

Some of the synthesized compounds were tested [44] for their antiproliferative activity in A2058 melanoma cells and proved to exert a modest to good activity (Figure 2). The best results were obtained with the 4-(trifluoromethyl)benzaldehyde hydrazone $\mathbf{2 u}$ and the 8-benzylamino pyrido[2,3- $b$ ]pyrazine 2o which induced $\sim 64 \%$ growth inhibition at $10^{-5} \mathrm{M}$.

Compounds $\mathbf{1} \mathbf{c}-\mathbf{e}, \mathbf{1 g}, \mathbf{2} \mathbf{d}-\mathbf{g}, \mathbf{2} \mathbf{i}-\mathbf{v}$ and $\mathbf{3 h}$ were evaluated [44] against a short panel of disease-relevant protein kinases. Protein kinases are drug targets often deregulated in diseases such as cancers and neurodegenerative disorders [45]. No significant inhibition of the following kinases was observed: Cyclin-dependent kinases 2 (CDK2/Cyclin A), 5 (CDK5/p25) and 9 (CDK9/Cyclin T), proto-oncogene kinase PIM1, CDC2-like kinase 1 (CLK1), dual specificity tyrosine phosphorylation regulated kinase 1A (DYRK1A), glycogen-synthase kinase 3 (GSK3; $\alpha / \beta$ or $\beta$ ), casein kinase 1 (CK1; $\delta / \varepsilon$ or $\varepsilon$ ), and mitotic kinase Haspin). Table S1 in Supplementary Materials shows the results obtained. 


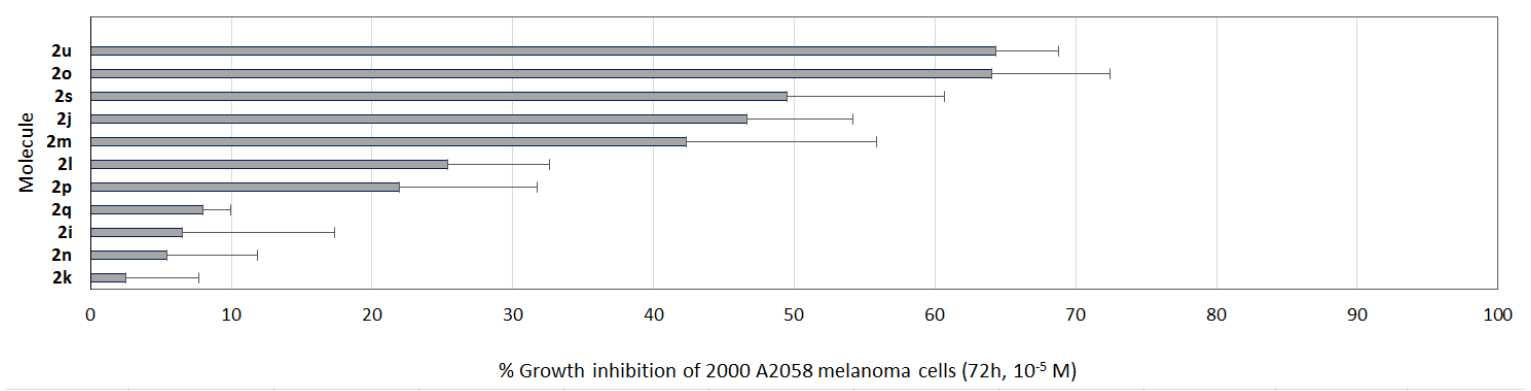

Figure 2. Antiproliferative activity of some of the synthesized compounds at $10^{-5} \mathrm{M}$ after $72 \mathrm{~h}$ in A2058 human melanoma cells.

\section{Materials and Methods}

\subsection{General Information}

All the reactions were performed under a dry argon atmosphere. THF was distilled over sodium/benzophenone. Column chromatography separations were achieved on silica gel $(40-63 \mu \mathrm{m})$. Melting points were measured on a Kofler apparatus. IR spectra were taken on an ATR Spectrum 100 spectrometer (Perkin-Elmer). ${ }^{1} \mathrm{H}$ - and ${ }^{13} \mathrm{C}$-Nuclear Magnetic Resonance (NMR) spectra were recorded either on an Avance III spectrometer (291 K) at $300 \mathrm{MHz}$ and $75 \mathrm{MHz}$, respectively, or on an Avance III HD spectrometer (298 K) at $500 \mathrm{MHz}$ and $126 \mathrm{MHz}$, respectively (Bruker, Billevica, Massachussets, USA). ${ }^{1} \mathrm{H}$ chemical shifts $(\delta)$ are given in ppm relative to the solvent residual peak and ${ }^{13} \mathrm{C}$ chemical shifts are relative to the central peak of the solvent signal [46]. 2,3-Diphenylpyrido[2,3-b]pyrazine (2a) [6], 8-bromo-2,3-diphenylpyrido[3,4-b]pyrazine (3a) [25,26] and 7-bromo-2,3-diphenylpyrido[2,3-b] pyrazine (4a) [6] were prepared as reported previously. The biological activity assays were performed as reported previously [44].

\subsection{Crystallography}

The X-ray diffraction data were collected either using an APEXII Bruker-AXS diffractometer (graphite monochromatized Mo-K $\alpha$ radiation $(\lambda=0.71073 \AA)$ ) for the compounds $\mathbf{1} \mathbf{b}^{\prime}$ and $\mathbf{2} \mathbf{i}$, or using a D8 VENTURE Bruker AXS diffractometer (multilayer monochromatized Mo-K $\alpha$ radiation $(\lambda=0.71073$ $\AA$ )) equipped with a (CMOS) PHOTON 100 detector for $\mathbf{2 f}, \mathbf{2} \mathbf{p}, \mathbf{3 h}$ and $\mathbf{2 d}$, at the temperature given in the crystal data. For $\mathbf{1} \mathbf{b}^{\prime}$ and $\mathbf{2} \mathbf{i}$, the structure was solved by direct methods using SIR97 [47]. For $\mathbf{2 f}$, $\mathbf{2 p}$, $\mathbf{3 h}$ and $\mathbf{2 d}$, they were solved by dual-space algorithm using the SHELXT program [48]. Structural refinements were performed with full-matrix least-square methods based on $F^{2}$ (SHELXL) [49]. In the case of $\mathbf{2 f}$ and $\mathbf{3 h}$, the contribution of the disordered solvents to the calculated structure factors was estimated following the BYPASS algorithm [50], implemented as the SQUEEZE option in PLATON [51]; a new data set, free of solvent contribution, was then used in the final refinement. All non-hydrogen atoms were refined with anisotropic atomic displacement parameters. Except nitrogen linked hydrogen atom that was introduced in the structural model through Fourier difference maps analysis (2f, $\mathbf{2} \mathbf{p}$, 3h), $\mathrm{H}$ atoms were finally included in their calculated positions and treated as riding on their parent atom with constrained thermal parameters. The molecular diagrams were generated by ORTEP-3 (version 2.02) [52].

\subsection{Deprotometalation Followed by Trapping with Electrophiles}

\subsubsection{General Procedure 1}

To a solution of 2,2,6,6-tetramethylpiperidine $(0.51 \mathrm{~mL}, 3.0 \mathrm{mmol})$ in THF $(3 \mathrm{~mL})$ at $0{ }^{\circ} \mathrm{C}$ were successively added BuLi (about $1.6 \mathrm{M}$ hexanes solution, $3.0 \mathrm{mmol}$ ) and, $15 \mathrm{~min}$ later, $\mathrm{ZnCl}_{2}$. TMEDA [53] $(0.25 \mathrm{~g}, 1.0 \mathrm{mmol})$. After $15 \mathrm{~min}$ at $0{ }^{\circ} \mathrm{C}$, the pyrazine $(2.0 \mathrm{mmol})$ was introduced, and the mixture was stirred for $2 \mathrm{~h}$ at $\mathrm{rt}$ before addition of $\mathrm{I}_{2}(0.76 \mathrm{~g}, 3.0 \mathrm{mmol})$ in THF $(3 \mathrm{~mL})$ at $0{ }^{\circ} \mathrm{C}$. The mixture was 
stirred at this temperature for $1 \mathrm{~h}$ before addition of an aqueous saturated solution of $\mathrm{Na}_{2} \mathrm{~S}_{2} \mathrm{O}_{3}(10 \mathrm{~mL})$ and extraction with EtOAc $(3 \times 20 \mathrm{~mL})$. The combined organic layers were dried over $\mathrm{MgSO}_{4}$, filtered and concentrated under reduced pressure. The crude product was purified by chromatography over silica gel (the eluent is given in the product description).

\subsubsection{5-Iodo-2,3-diphenylquinoxaline (1b)}

The general procedure 1 using 2,3-diphenylquinoxaline (1a, $0.56 \mathrm{~g})$ gave $\mathbf{1 b}$ (eluent: heptane$\left.\mathrm{CH}_{2} \mathrm{Cl}_{2} 60: 40 ; \mathrm{R}_{\mathrm{f}}=0.55\right)$ in $74 \%$ yield as a pale yellow powder. Mp: $148{ }^{\circ} \mathrm{C}$. IR: $486,529,551,602,689$, 695, 701, 763, 776, 796, 892, 978, 1023, 1068, 1079, 1184, 1281, 1336, 1384, 1497, 1534, $3051 \mathrm{~cm}^{-1} .{ }^{1} \mathrm{H}-\mathrm{NMR}$ $\left(\mathrm{CDCl}_{3}\right): 7.31-7.41(\mathrm{~m}, 6 \mathrm{H}), 7.48(\mathrm{dd}, 1 \mathrm{H}, J=8.3$ and $7.4 \mathrm{~Hz}), 7.54-7.57(\mathrm{~m}, 2 \mathrm{H}), 7.64-7.67(\mathrm{~m}, 2 \mathrm{H})$, $8.15(\mathrm{dd}, 1 \mathrm{H}, J=8.4$ and $1.3 \mathrm{~Hz}), 8.36(\mathrm{dd}, 1 \mathrm{H}, J=7.4$ and $1.3 \mathrm{~Hz}) \cdot{ }^{13} \mathrm{C}-\mathrm{NMR}\left(\mathrm{CDCl}_{3}\right): 102.8(\mathrm{C}), 128.3$ $(2 \mathrm{CH}), 128.5(2 \mathrm{CH}), 129.2(\mathrm{CH}), 129.3(\mathrm{CH}), 129.9(2 \mathrm{CH}), 130.0(\mathrm{CH}), 130.5(2 \mathrm{CH}), 131.0(\mathrm{CH}), 138.2(\mathrm{C})$, $138.7(\mathrm{C}), 140.1(\mathrm{CH}), 140.9$ (C), $141.3(\mathrm{C}), 153.9$ (C), 154.1 (C). Anal. Calc. for $\mathrm{C}_{20} \mathrm{H}_{13} \mathrm{IN}_{2}$ (408.24): C 58.84, H 3.21, N , 6.86. Found: C 59.05, H 3.27, N, 6.70. 5,8-Diiodo-2,3-diphenylquinoxaline (1 $\left.\mathbf{b}^{\prime}\right)$ was similarly isolated (eluent: heptane- $\mathrm{CH}_{2} \mathrm{Cl}_{2} 60: 40 ; \mathrm{R}_{\mathrm{f}}=0.69$ ) in $7 \%$ yield as a yellow powder. Mp: 222 ${ }^{\circ} \mathrm{C}$. IR: 533, 572, 613, 649, 692, 771, 824, 893, 978, 1025, 1055, 1077, 1169, 1209, 1325, 1383, 1447, 2930, $3059 \mathrm{~cm}^{-1} .{ }^{1} \mathrm{H}-\mathrm{NMR}\left(\mathrm{CDCl}_{3}\right)$ : 7.34-7.45 (m, 6H), 7.64-7.76 (m, 4H), $8.02(\mathrm{~s}, 2 \mathrm{H}) .{ }^{13} \mathrm{C}-\mathrm{NMR}\left(\mathrm{CDCl}_{3}\right)$ : $103.5(2 \mathrm{C}), 128.4(4 \mathrm{CH}), 129.7(2 \mathrm{CH}), 130.4(4 \mathrm{CH}), 137.7(2 \mathrm{C}), 140.6(2 \mathrm{CH}), 140.8(2 \mathrm{C}), 154.5(2 \mathrm{C})$. Crystal data for $\mathbf{1 b}^{\prime} . \mathrm{C}_{20} \mathrm{H}_{12} \mathrm{I}_{2} \mathrm{~N}_{2}, M=534.12, T=150(2) \mathrm{K}$, monoclinic, $P 22_{1}, a=10.1153(9), b=5.8725(5)$, $c=14.9603(14) \AA, \beta=98.489(4)^{\circ}, V=878.94(14) \AA^{3}, Z=2, d=2.018 \mathrm{~g} \mathrm{~cm}^{-3}, \mu=3.581 \mathrm{~mm}^{-1}$. A final refinement on $F^{2}$ with 3888 unique intensities and 217 parameters converged at $\omega R\left(F^{2}\right)=0.0701(R(F)$ $=0.0343$ ) for 3602 observed reflections with $I>2 \sigma(I)$. CCDC 1858478 .

\subsubsection{8-Iodo-2,3-diphenylpyrido[2,3-b]pyrazine (2b-I)}

The general procedure 1 using 2,3-diphenylpyrido[2,3-b]pyrazine (2a, $0.57 \mathrm{~g}$ ) gave $\mathbf{2 b}$-I (eluent: $\left.\mathrm{CH}_{2} \mathrm{Cl}_{2} ; \mathrm{R}_{\mathrm{f}}=0.34\right)$ in $70 \%$ yield as a whitish powder. Mp: $220^{\circ} \mathrm{C}$. IR: $534,562,613,624,637,699$, 980, 1023, 1336, 1416, 1519, 1570, $3068 \mathrm{~cm}^{-1} .{ }^{1} \mathrm{H}-\mathrm{NMR}\left(\mathrm{CDCl}_{3}\right): 7.32-7.44(\mathrm{~m}, 6 \mathrm{H}), 7.64-7.69(\mathrm{~m}, 4 \mathrm{H})$, $8.28(\mathrm{~d}, 1 \mathrm{H}, J=4.5 \mathrm{~Hz}), 8.70(\mathrm{~d}, 1 \mathrm{H}, J=4.6 \mathrm{~Hz}) .{ }^{13} \mathrm{C}-\mathrm{NMR}\left(\mathrm{CDCl}_{3}\right): 116.1(\mathrm{C}), 128.3(2 \mathrm{CH}), 128.4(2 \mathrm{CH})$, $129.7(\mathrm{CH}), 129.8(\mathrm{CH}), 130.3(2 \mathrm{CH}), 130.3(2 \mathrm{CH}), 135.6(\mathrm{CH}), 136.6(\mathrm{C}), 137.6(\mathrm{C}), 137.6(\mathrm{C}), 149.1(\mathrm{C})$, 153.6 (CH), $155.0(\mathrm{C}), 157.1(\mathrm{C})$. Anal. Calc. for $\mathrm{C}_{19} \mathrm{H}_{12} \mathrm{IN}_{3}$ (409.23): C 55.77, H 2.96, N, 10.27. Found: $\mathrm{C}$ 55.91, H 3.06, N, 10.03 .

\subsubsection{8-Bromo-2,3-diphenylpyrido[2,3-b]pyrazine (2b-Br)}

To a stirred mixture of 2,3-diphenyl pyrido[2,3-b]pyrazine (2a, $0.28 \mathrm{~g}, 1.0 \mathrm{mmol})$ and $\mathrm{ZnCl}_{2}$. TMEDA [53] ( $0.26 \mathrm{~g}, 1.0 \mathrm{mmol})$ in THF $(1 \mathrm{~mL})$ at $-20{ }^{\circ} \mathrm{C}$ was added dropwise a solution of LiTMP (prepared by adding BuLi (about $1.6 \mathrm{M}$ hexanes solution, $1.2 \mathrm{mmol}$ ) to a stirred, cooled $\left(-20{ }^{\circ} \mathrm{C}\right.$ ) solution of 2,2,6,6-tetramethylpiperidine $(0.24 \mathrm{~mL}, 1.2 \mathrm{mmol})$ in THF $(2 \mathrm{~mL})$ and stirring for $15 \mathrm{~min})$ cooled at $-20^{\circ} \mathrm{C}$. After $30 \mathrm{~min}$ at $-20^{\circ} \mathrm{C}, \mathrm{Br}_{2}(97 \mu \mathrm{L}, 2.0 \mathrm{mmol})$ was introduced, and the mixture was stirred for $1 \mathrm{~h}$ before addition of an aqueous saturated solution of $\mathrm{Na}_{2} \mathrm{~S}_{2} \mathrm{O}_{3}(5 \mathrm{~mL})$ and extraction with EtOAc $(3 \times 20 \mathrm{~mL})$. The combined organic layers were dried over $\mathrm{MgSO}_{4}$, filtered and concentrated under reduced pressure. The crude product was purified by chromatography over silica gel (eluent: $\mathrm{CH}_{2} \mathrm{Cl}_{2}$-EtOAc 90:10; $\mathrm{R}_{\mathrm{f}}=0.50$ ) to give $\mathbf{2 b -} \mathbf{B r}$ in $60 \%$ yield as a whitish powder. Mp: $183{ }^{\circ} \mathrm{C}$. IR: 491 , 538, 563, 615, 625, 649, 698, 767, 839, 985, 1021, 1049, 1090, 1179, 1241, 1336, 1387, 1421, 1460, 1524, 1584, $3067 \mathrm{~cm}^{-1} .{ }^{1} \mathrm{H}-\mathrm{NMR}\left(\mathrm{CDCl}_{3}\right): 7.32-7.42(\mathrm{~m}, 6 \mathrm{H}), 7.63-7.66(\mathrm{~m}, 4 \mathrm{H}), 8.00(\mathrm{~d}, 1 \mathrm{H}, J=4.7 \mathrm{~Hz}), 8.91(\mathrm{~d}, 1 \mathrm{H}$, $J=4.7 \mathrm{~Hz}) .{ }^{13} \mathrm{C}-\mathrm{NMR}\left(\mathrm{CDCl}_{3}\right): 128.3(\mathrm{CH}), 128.4(\mathrm{CH}), 128.7(\mathrm{CH}), 129.7(\mathrm{CH}), 129.8(\mathrm{CH}), 130.3(\mathrm{CH})$, $130.3(\mathrm{CH}), 134.7(\mathrm{C}), 136.3(\mathrm{C}), 137.7(\mathrm{C}), 137.9$ (C), $150.1(\mathrm{C}), 153.4(\mathrm{CH}), 154.9(\mathrm{C}), 157.0(\mathrm{C})$. Anal. Calc. for $\mathrm{C}_{19} \mathrm{H}_{12} \mathrm{BrN}_{3}$ (362.23): C 63.00, H 3.34, N, 11.60. Found: C 63.24, H 3.58, N, 11.43. 


\subsubsection{8-Chloro-2,3-diphenylpyrido[2,3-b]pyrazine (2b-Cl)}

To a stirred mixture of 2,3-diphenyl pyrido[2,3-b]pyrazine $(2 \mathrm{a}, 0.28 \mathrm{~g}, 1.0 \mathrm{mmol})$ and $\mathrm{ZnCl}_{2}$. TMEDA [53] $(0.26 \mathrm{~g}, 1.0 \mathrm{mmol})$ in THF $(1 \mathrm{~mL})$ at $-20^{\circ} \mathrm{C}$ was added dropwise a solution of LiTMP (prepared by adding BuLi (about $1.6 \mathrm{M}$ hexanes solution, $1.2 \mathrm{mmol}$ ) to a stirred, cooled $\left(-20{ }^{\circ} \mathrm{C}\right.$ ) solution of 2,2,6,6-tetramethylpiperidine $(0.24 \mathrm{~mL}, 1.2 \mathrm{mmol})$ in THF $(2 \mathrm{~mL})$ and stirring for $15 \mathrm{~min})$ cooled at $-20^{\circ} \mathrm{C}$. After $30 \mathrm{~min}$ at $-20^{\circ} \mathrm{C}$, trichloroisocyanuric acid $(0.30 \mathrm{~g}, 1.3 \mathrm{mmol})$ was introduced (CAUTION: dissolution of trichloroisocyanuric acid in THF at a temperature above $0{ }^{\circ} \mathrm{C}$ produces intense heat), and the mixture was stirred at this temperature for $1 \mathrm{~h}$ before addition of water $(5 \mathrm{~mL})$ and extraction with EtOAc $(3 \times 20 \mathrm{~mL})$. The combined organic layers were dried over $\mathrm{MgSO}_{4}$, filtered and concentrated under reduced pressure. The crude product was purified by chromatography over silica gel (eluent: $\mathrm{CH}_{2} \mathrm{Cl}_{2}$-EtOAc 90:10; $\mathrm{R}_{\mathrm{f}}=0.60$ ) to give $2 \mathbf{b}-\mathrm{Cl}$ in $62 \%$ yield as a whitish powder. Mp: $180^{\circ} \mathrm{C}$. IR: 534, 544, 617, 658, 699, 770, 851, 991, 1025, 1055, 1193, 1242, 1341, 1388, 1422, 1442, 1452, 1532, 1583, 3034, $3051 \mathrm{~cm}^{-1} .{ }^{1} \mathrm{H}-\mathrm{NMR}\left(\mathrm{CDCl}_{3}\right): 7.31-7.44(\mathrm{~m}, 6 \mathrm{H}), 7.62-7.66(\mathrm{~m}, 4 \mathrm{H}), 7.79(\mathrm{~d}, 1 \mathrm{H}, J=4.7 \mathrm{~Hz})$, $9.02(\mathrm{~d}, 1 \mathrm{H}, J=4.7 \mathrm{~Hz}) .{ }^{13} \mathrm{C}-\mathrm{NMR}\left(\mathrm{CDCl}_{3}\right)$ : $125.1(\mathrm{CH}), 128.3(\mathrm{CH}), 128.5(\mathrm{CH}), 129.7(\mathrm{CH}), 129.8(\mathrm{CH})$, $130.2(\mathrm{CH}), 130.3(\mathrm{CH}), 133.7(\mathrm{C}), 137.8(\mathrm{C}), 138.1(\mathrm{C}), 144.5(\mathrm{C}), 150.5(\mathrm{C}), 153.3(\mathrm{CH}), 154.8(\mathrm{C}), 157.1$ (C). Anal. Calc. for $\mathrm{C}_{19} \mathrm{H}_{12} \mathrm{ClN}_{3}$ (317.78): C 71.81, H 3.81, N, 13.22. Found: C 71.77, H 3.85, N, 13.14 .

\subsubsection{General Procedure 2}

To a stirred mixture of the pyrazine $(1.0 \mathrm{mmol})$ and $\mathrm{ZnCl}_{2} \cdot$ TMEDA [53] $(0.26 \mathrm{~g}, 1.0 \mathrm{mmol})$ in THF $(1 \mathrm{~mL})$ at $-20^{\circ} \mathrm{C}$ was added dropwise a solution of LiTMP (prepared by adding BuLi (about $1.6 \mathrm{M}$ hexanes solution, $1.2 \mathrm{mmol})$ to a stirred, cooled $\left(-20^{\circ} \mathrm{C}\right)$ solution of 2,2,6,6-tetramethylpiperidine $(0.24 \mathrm{~mL}, 1.2 \mathrm{mmol})$ in THF $(2 \mathrm{~mL})$ and stirring for $15 \mathrm{~min})$ cooled at $-20^{\circ} \mathrm{C}$. After $30 \mathrm{~min}$ at $-20^{\circ} \mathrm{C}$, $\mathrm{I}_{2}(0.37 \mathrm{~g}, 1.5 \mathrm{mmol})$ in THF $(2 \mathrm{~mL})$ was introduced, and the mixture was stirred at this temperature for $1 \mathrm{~h}$ before addition of an aqueous saturated solution of $\mathrm{Na}_{2} \mathrm{~S}_{2} \mathrm{O}_{3}(5 \mathrm{~mL})$ and extraction with EtOAc $(3 \times 20 \mathrm{~mL})$. The combined organic layers were dried over $\mathrm{MgSO}_{4}$, filtered and concentrated under reduced pressure. The crude product was purified by chromatography over silica gel (the eluent is given in the product description).

\subsubsection{8-Bromo-7-iodo-2,3-diphenylpyrido[3,4-b]pyrazine ( $3 \mathbf{b})$}

The general procedure 2 using 8-bromo-2,3-diphenylpyrido[3,4-b]pyrazine (3a [25,26], $0.36 \mathrm{~g})$ gave $3 \mathbf{b}$ (eluent: $\mathrm{CH}_{2} \mathrm{Cl}_{2}$-petroleum ether $80: 20 ; \mathrm{R}_{\mathrm{f}}=0.44$ ) in $67 \%$ yield as a red powder. Mp: $186-188$ ${ }^{\circ} \mathrm{C}$. IR: 493, 529, 559, 600, 658, 695, 765, 984, 1025, 1055, 1117, 1238, 1315, 1373, 1399, 1446, 1493, 1551, 3034, $3060 \mathrm{~cm}^{-1}$. ${ }^{1} \mathrm{H}-\mathrm{NMR}\left(\mathrm{CDCl}_{3}\right): 7.34-7.47(\mathrm{~m}, 6 \mathrm{H}), 7.54-7.57(\mathrm{~m}, 2 \mathrm{H}), 7.62-7.65(\mathrm{~m}, 2 \mathrm{H}), 9.27$ $(\mathrm{s}, 1 \mathrm{H}) .{ }^{13} \mathrm{C}-\mathrm{NMR}\left(\mathrm{CDCl}_{3}\right): 121.7(\mathrm{C}), 128.6(2 \mathrm{CH}), 128.7(2 \mathrm{CH}), 129.7(\mathrm{C}), 129.8(2 \mathrm{CH}), 130.1(\mathrm{CH})$, $130.5(2 \mathrm{CH}), 130.5(\mathrm{CH}), 136.0(\mathrm{C}), 137.4(\mathrm{C}), 137.7(\mathrm{C}), 142.3(\mathrm{C}), 152.5(\mathrm{CH}), 156.2(\mathrm{C}), 158.6(\mathrm{C})$. Anal. Calc. for $\mathrm{C}_{19} \mathrm{H}_{11} \mathrm{BrIN}_{3}$ (488.13): C 46.75, H 2.27, N, 8.61. Found: C 46.89, H 2.49, N, 8.55. 8-Bromo-5,7-diiodo-2,3-diphenyl pyrido[3,4- $b$ ]pyrazine, also formed in $<5 \%$ yield, was identified by its ${ }^{1} \mathrm{H}-\mathrm{NMR}\left(\mathrm{CDCl}_{3}\right):$ 7.36-7.47 (m, 6H), 7.54-7.57 (m, 2H), 7.65-7.68 (m, 4H).

\subsubsection{7-Bromo-6-iodo-2,3-diphenylpyrido[2,3-b]pyrazine (4b)}

The general procedure 2 using 7-bromo-2,3-diphenylpyrido[2,3-b]pyrazine (4a [54], prepared in $90 \%$ yield [6], 0.36 g) gave $4 \mathbf{b}$ (eluent: $\mathrm{CH}_{2} \mathrm{Cl}_{2}$-heptane 70:30; $\mathrm{R}_{\mathrm{f}}$ (heptane- $\mathrm{CH}_{2} \mathrm{Cl}_{2}$ 80:20) $=0.80$ ) in $5 \%$ yield as a yellow powder. Mp: 150-152 ${ }^{\circ} \mathrm{C}$. IR: 495, 547, 596, 615, 697, 731, 770, 778, 903, 937, 1025, 1060, 1107, 1178, 1274, 1332, 1390, 1448, 1502, 1562, 1603, 1699, 1768, 2734, 2940, $3064 \mathrm{~cm}^{-1} .{ }^{1} \mathrm{H}-\mathrm{NMR}$ $\left(\mathrm{CDCl}_{3}\right): 7.30-7.43(\mathrm{~m}, 6 \mathrm{H}), 7.52-7.55(\mathrm{~m}, 2 \mathrm{H}), 7.59-7.62(\mathrm{~m}, 2 \mathrm{H}), 8.62(\mathrm{~s}, 1 \mathrm{H}) .{ }^{13} \mathrm{C}-\mathrm{NMR}\left(\mathrm{CDCl}_{3}\right): 128.3$ $(2 \mathrm{CH}), 128.6(2 \mathrm{CH}), 128.7(\mathrm{C}), 129.9(\mathrm{CH}), 129.9(2 \mathrm{CH}), 130.0(\mathrm{CH}), 130.0(\mathrm{C}), 130.3(2 \mathrm{CH}), 135.5(\mathrm{C})$, $137.6(\mathrm{C}), 138.0(\mathrm{C}), 139.5(\mathrm{CH}), 148.0(\mathrm{C}), 155.9$ (C), 157.1 (C). Anal. Calc. for $\mathrm{C}_{19} \mathrm{H}_{11} \mathrm{BrIN}_{3}$ (488.13): C 46.75, H 2.27, N, 8.61. Found: C 46.93, H 2.38, N, 8.49. 


\subsection{Suzuki Coupling Reactions}

\subsubsection{General Procedure 3}

To a stirred mixture of the iodide $(0.50 \mathrm{mmol})$ and $\mathrm{Pd}\left(\mathrm{PPh}_{3}\right)_{4}(29 \mathrm{mg}, 25 \mu \mathrm{mol})$ in degassed 1,2-dimethoxyethane $(5 \mathrm{~mL})$ was added the boronic acid $(0.60 \mathrm{mmol})$ and $\mathrm{NaHCO}_{3}(2.0 \mathrm{mmol})$ in degassed water $(1.6 \mathrm{~mL})$. The resulting mixture was heated at $80^{\circ} \mathrm{C}$ for $3 \mathrm{~h}$ and cooled to $\mathrm{rt}$ before addition of water $(5 \mathrm{~mL})$ and extraction with EtOAc $(3 \times 10 \mathrm{~mL})$. The combined organic layers were washed with brine $(10 \mathrm{~mL})$, dried over $\mathrm{MgSO}_{4}$, filtered and concentrated under reduced pressure. The crude product was purified by chromatography over silica gel (the eluent is given in the product description).

\subsubsection{2,3,5-Triphenylquinoxaline (1c)}

The general procedure 3 using 5-iodo-2,3-diphenyl quinoxaline (1b, $0.20 \mathrm{~g})$ and phenylboronic acid (73 mg) gave 1c (eluent: $\mathrm{CH}_{2} \mathrm{Cl}_{2}$-heptane 60:40; $\mathrm{R}_{\mathrm{f}}=0.35$ ) in $42 \%$ yield as a white powder. Mp: $150{ }^{\circ} \mathrm{C}$. IR: 763, 804, 841, 927, 984, 1023, 1081, 1128, 1233, 1336, 1388, 1433, 1444, 1491, 1566, 2858, 2927, 2965, $3064 \mathrm{~cm}^{-1} .{ }^{1} \mathrm{H}-\mathrm{NMR}\left(\mathrm{CDCl}_{3}\right):$ 7.26-7.34 (m, 3H), 7.36-7.48 (m, 4H), 7.52-7.64 (m, 6H), 7.81-7.89 $(\mathrm{m}, 4 \mathrm{H}), 8.21(\mathrm{dd}, 1 \mathrm{H}, J=7.3$ and $2.5 \mathrm{~Hz}) .{ }^{13} \mathrm{C}-\mathrm{NMR}\left(\mathrm{CDCl}_{3}\right): 127.7(\mathrm{CH}), 128.0(2 \mathrm{CH})$, $128.1(2 \mathrm{CH}), 128.5(2 \mathrm{CH}), 128.7(\mathrm{CH}), 128.8(\mathrm{CH}), 129.0(\mathrm{CH}), 129.8(\mathrm{CH}), 129.9(2 \mathrm{CH}), 130.3(2 \mathrm{CH})$, $130.4(\mathrm{CH}), 131.1(2 \mathrm{CH}), 138.4(\mathrm{C}), 139.0(\mathrm{C}), 139.1(\mathrm{C}), 139.4(\mathrm{C}), 140.6(\mathrm{C}), 141.3(\mathrm{C}), 152.4(\mathrm{C}), 152.9(\mathrm{C})$. Anal. Calc. for $\mathrm{C}_{26} \mathrm{H}_{18} \mathrm{~N}_{2}$ (358.44): C 87.12, H 5.06, N, 7.82. Found: C 87.25, H 5.22, N, 7.70.

\subsubsection{2,3-Diphenyl-5-(2-thienyl)quinoxaline (1d)}

The general procedure 3 using 5-iodo-2,3-diphenylquinoxaline (1b, $0.20 \mathrm{~g})$ and 2-thienylboronic acid (77 mg) gave 1d (eluent: $\mathrm{CH}_{2} \mathrm{Cl}_{2}$-heptane 60:40; $\mathrm{R}_{\mathrm{f}}=0.20$ ) in $97 \%$ yield as a yellow powder. Mp: $210{ }^{\circ} \mathrm{C}$. IR: 738, 766, 796, 828, 854, 916, 933, 969, 1025, 1053, 1083, 1163, 1238, 1336, 1390, 1442, 1495, 1562, 1592, $3064 \mathrm{~cm}^{-1} .{ }^{1} \mathrm{H}-\mathrm{NMR}\left(\mathrm{CDCl}_{3}\right): 7.18(\mathrm{dd}, 1 \mathrm{H}, J=5.1$ and $3.7 \mathrm{~Hz}), 7.32-7.40(\mathrm{~m}, 6 \mathrm{H})$, $7.51(\mathrm{dd}, 1 \mathrm{H}, J=5.1$ and $1.2 \mathrm{~Hz}), 7.58-7.61(\mathrm{~m}, 2 \mathrm{H}), 7.67-7.70(\mathrm{~m}, 2 \mathrm{H}), 7.76(\mathrm{dd}, 1 \mathrm{H}, J=8.3$ and $7.4 \mathrm{~Hz})$, $7.88(\mathrm{dd}, 1 \mathrm{H}, J=3.7$ and $1.2 \mathrm{~Hz}), 8.08(\mathrm{dd}, 1 \mathrm{H}, J=8.3$ and $1.3 \mathrm{~Hz}), 8.13(\mathrm{dd}, 1 \mathrm{H}, J=7.4$ and $1.3 \mathrm{~Hz})$. ${ }^{13} \mathrm{C}-\mathrm{NMR}\left(\mathrm{CDCl}_{3}\right)$ : $126.7(\mathrm{CH}), 126.9(\mathrm{CH}), 127.5(\mathrm{CH}), 128.1(\mathrm{CH}), 128.2(2 \mathrm{CH}), 128.5(2 \mathrm{CH}), 128.8(\mathrm{CH})$, 129.0 (CH), $129.0(\mathrm{CH}), 129.9(2 \mathrm{CH}), 129.9(\mathrm{CH}), 130.6(2 \mathrm{CH}), 133.0(\mathrm{C}), 137.6(\mathrm{C}), 138.8(\mathrm{C}), 138.9(\mathrm{C})$, 139.2 (C), $141.4(\mathrm{C}), 152.3$ (C), 153.2 (C). Anal. Calc. for $\mathrm{C}_{24} \mathrm{H}_{16} \mathrm{~N}_{2} \mathrm{~S}$ (364.47): C 79.09, H 4.43, N, 7.69. Found: C 79.11, H 4.48, N, 7.72 .

\subsubsection{2,3-Diphenyl-8-(2-thienyl)pyrido[2,3-b]pyrazine (2d)}

The general procedure 3 using 8-iodo-2,3-diphenylpyrido[2,3-b]pyrazine (2b-I, $0.20 \mathrm{~g})$ and 2-thienylboronic acid (77 mg) gave 2d (eluent: $\mathrm{CH}_{2} \mathrm{Cl}_{2}$-EtOAc 95:5; $\mathrm{R}_{\mathrm{f}}=0.50$ ) in $75 \%$ yield as a pale yellow powder. Mp: $215^{\circ} \mathrm{C}$. IR: 540, 695, 744, 1025, 1096, 1120, 1188, 1238, 1336, 1384, 1435, 1480, 551, 1568, 2927, 2965, $3060 \mathrm{~cm}^{-1} .{ }^{1} \mathrm{H}-\mathrm{NMR}\left(\mathrm{CDCl}_{3}\right): 7.23(\mathrm{dd}, 1 \mathrm{H}, J=5.1$ and $3.8 \mathrm{~Hz}), 7.32-7.45(\mathrm{~m}, 6 \mathrm{H})$, 7.66-7.72 (m, 5H), $7.98(\mathrm{~d}, 1 \mathrm{H}, J=4.8 \mathrm{~Hz}), 8.10(\mathrm{dd}, 1 \mathrm{H}, J=3.8$ and $1.2 \mathrm{~Hz}), 9.10(\mathrm{~d}, 1 \mathrm{H}, J=4.8 \mathrm{~Hz})$. ${ }^{13} \mathrm{C}-\mathrm{NMR}\left(\mathrm{CDCl}_{3}\right)$ : $120.3(\mathrm{CH}), 127.2(\mathrm{CH}), 128.3(2 \mathrm{CH}), 128.4(2 \mathrm{CH}), 129.2(\mathrm{CH}), 129.4(\mathrm{CH}), 129.5$ (CH), $130.3(2 \mathrm{CH}), 130.5(2 \mathrm{CH}), 132.4(\mathrm{CH}), 132.4(\mathrm{C}), 136.0(\mathrm{C}), 138.2(\mathrm{C}), 138.3(\mathrm{C}), 140.9(\mathrm{C}), 150.2(\mathrm{C})$, $153.1(\mathrm{C}), 153.9(\mathrm{CH}), 155.8(\mathrm{C})$. Crystal data for 2 d. $\mathrm{C}_{23} \mathrm{H}_{15} \mathrm{~N}_{3} \mathrm{~S}, M=365.44, \mathrm{~T}=150(2) \mathrm{K}$, triclinic, $P 1$, $a=6.6311(18), b=9.939(3), c=13.655(4) \AA, \alpha=81.914(12), \beta=80.405(11), \gamma=89.955(10)^{\circ}, V=878.3(4)$ $\AA^{3}, Z=2, d=1.382 \mathrm{~g} \mathrm{~cm}^{-3}, \mu=0.197 \mathrm{~mm}^{-1}$. A final refinement on $F^{2}$ with 7113 unique intensities and 236 parameters converged at $\omega R\left(F^{2}\right)=0.3351(R(F)=0.1327)$ for 6147 observed reflections with $I>2 \sigma(I)$. CCDC 1858479. 


\subsubsection{5-(2-Aminophenyl)-2,3-diphenylquinoxaline (1e)}

The general procedure 3 using 5-iodo-2,3-diphenylquinoxaline (1b, $0.20 \mathrm{~g})$ and 2-aminophenylboronic acid (82 mg) gave 1e (eluent: heptane- $\mathrm{CH}_{2} \mathrm{Cl}_{2} 70: 30 ; \mathrm{R}_{\mathrm{f}}=0.31$ ) in $92 \%$ yield as a yellow powder. Mp: $178^{\circ} \mathrm{C}$. IR: 689, 702, 740, 771, 977, 1307, 1342, 1492, 1626, 3025, 3060, 3212, 3328, $3468 \mathrm{~cm}^{-1}$. ${ }^{1} \mathrm{H}-\mathrm{NMR}\left(\mathrm{CDCl}_{3}\right): 3.87\left(\mathrm{br} \mathrm{s}, 2 \mathrm{H}, \mathrm{NH}_{2}\right), 6.85(\mathrm{dd}, 1 \mathrm{H}, J=7.9$ and $1.1 \mathrm{~Hz}), 6.92(\mathrm{td}, 1 \mathrm{H}$, $J=7.4$ and $1.2 \mathrm{~Hz}), 7.21-7.30(\mathrm{~m}, 5 \mathrm{H}), 7.35-7.40(\mathrm{~m}, 3 \mathrm{H}), 7.47-7.50(\mathrm{~m}, 2 \mathrm{H}), 7.55-7.58(\mathrm{~m}, 2 \mathrm{H}), 7.78-7.86$ $(\mathrm{m}, 2 \mathrm{H}), 8.20(\mathrm{dd}, 1 \mathrm{H}, J=7.8$ and $2.1 \mathrm{~Hz}) .{ }^{13} \mathrm{C}-\mathrm{NMR}\left(\mathrm{CDCl}_{3}\right): 116.5(\mathrm{CH}), 118.8(\mathrm{CH}), 125.7(\mathrm{C})$, $128.1(2 \mathrm{CH}), 128.5(2 \mathrm{CH}), 128.9(\mathrm{CH}), 129.0(\mathrm{CH}), 129.0(\mathrm{CH}), 129.0(\mathrm{CH}), 129.9(2 \mathrm{CH}), 130.2(\mathrm{CH})$, $130.3(2 \mathrm{CH}), 132.0(\mathrm{CH}), 132.3(\mathrm{CH}), 138.8(\mathrm{C}), 139.2(\mathrm{C}), 139.3(\mathrm{C}), 139.6(\mathrm{C}), 141.3(\mathrm{C}), 145.0(\mathrm{C})$, 152.5 (C), 153.2 (C). Anal. Calc. for $\mathrm{C}_{26} \mathrm{H}_{19} \mathrm{~N}_{3}$ (373.46): C 83.62, H 5.13, N, 11.25. Found: C 83.81, H 5.26, $\mathrm{N}, 11.17$.

\subsubsection{8-(2-Aminophenyl)-2,3-diphenylpyrido[2,3-b]pyrazine (2e)}

The general procedure 3 using 8-iodo-2,3-diphenylpyrido[2,3-b]pyrazine (2b-I, $0.20 \mathrm{~g})$ and 2-aminophenylboronic acid (82 mg) gave 2e (eluent: $\mathrm{CH}_{2} \mathrm{Cl}_{2}$-EtOAc 70:30; $\mathrm{R}_{\mathrm{f}}=0.50$ ) in 73\% yield as a yellow powder. Mp: $205^{\circ} \mathrm{C}$. IR: 687, 742, 766, 854, 981, 1015, 1047, 1237, 1307, 1382, 1489, 1623, 3024, 3055, $3345 \mathrm{~cm}^{-1} .{ }^{1} \mathrm{H}-\mathrm{NMR}\left(\mathrm{CDCl}_{3}\right): 3.99\left(\mathrm{br} \mathrm{s}, 2 \mathrm{H}, \mathrm{NH}_{2}\right), 6.87(\mathrm{dd}, 1 \mathrm{H}, J=8.4$ and $1.2 \mathrm{~Hz})$, $6.94(\mathrm{td}, 1 \mathrm{H}, J=7.4$ and $1.2 \mathrm{~Hz}), 7.25-7.40(\mathrm{~m}, 8 \mathrm{H}), 7.51-7.54(\mathrm{~m}, 2 \mathrm{H}), 7.65-7.68(\mathrm{~m}, 2 \mathrm{H}), 7.73(\mathrm{~d}, 1 \mathrm{H}$, $J=4.5 \mathrm{~Hz}), 9.19(\mathrm{~d}, 1 \mathrm{H}, J=4.4 \mathrm{~Hz}) .{ }^{13} \mathrm{C}-\mathrm{NMR}\left(\mathrm{CDCl}_{3}\right): 116.9(\mathrm{CH}), 118.7(\mathrm{CH}), 122.6(\mathrm{C}), 126.3(\mathrm{CH})$, $128.2(2 \mathrm{CH}), 128.2(2 \mathrm{CH}), 129.3(\mathrm{CH}), 129.5(\mathrm{CH}), 130.1(\mathrm{CH}), 130.1(2 \mathrm{CH}), 130.1(2 \mathrm{CH}), 132.0(\mathrm{CH})$, 134.3 (C), 138.1 (C), 138.2 (C), 144.9 (C), 149.0 (C), 149.8 (C), 153.4 (C), 154.1 (CH), 155.7 (C). Anal. Calc. for $\mathrm{C}_{25} \mathrm{H}_{18} \mathrm{~N}_{4}$ (374.45): C 80.19, H 4.85, N, 14.96. Found: C 80.07, H 4.87, N, 14.85.

\subsubsection{2,3-Diphenyl-11H-pyrazino[ $\left[2^{\prime}, 3^{\prime}: 4,5\right]$ pyrido[2,3-d]indole (3h)}

In a tube containing a stirred mixture of 8-bromo-7-iodo-2,3-diphenylpyrido[3,4- $b]$ pyrazine ( $3 \mathbf{b}$, $0.24 \mathrm{~g}, 0.50 \mathrm{mmol})$ and $\mathrm{Pd}\left(\mathrm{PPh}_{3}\right)_{4}(29 \mathrm{mg}, 25 \mu \mathrm{mol})$ in degassed 1,2-dimethoxyethane (5 mL) was introduced 2-aminophenylboronic acid $(82 \mathrm{mg}, 0.60 \mathrm{mmol})$ and $\mathrm{Na}_{2} \mathrm{CO}_{3}(2.0 \mathrm{mmol})$ in degassed water $(1.6 \mathrm{~mL})$. The sealed tube was heated overnight at $140{ }^{\circ} \mathrm{C}$ and cooled to $\mathrm{rt}$ before addition of saturated aqueous $\mathrm{NaHCO}_{3}(5 \mathrm{~mL})$ and extraction with EtOAc $(3 \times 10 \mathrm{~mL})$. The combined organic layers were washed with brine $(10 \mathrm{~mL})$, dried over $\mathrm{MgSO}_{4}$, filtered and concentrated under reduced pressure. The crude product was purified by chromatography over silica gel (eluent: $\mathrm{CH}_{2} \mathrm{Cl}_{2}-\mathrm{EtOAc}$ 90:10; $R_{\mathrm{f}}=0.28$ ) to give $3 \mathrm{~h}$ in $65 \%$ yield as a yellow powder. Mp: $284-286^{\circ} \mathrm{C}$. IR: $695,748,763,1025$, 1092, 1190, 1236, 1315, 1328, 1336, 1376, 1446, 1495, 1540, 1624, 3034, 3064, $3420 \mathrm{~cm}^{-1}$. ${ }^{1} \mathrm{H}-\mathrm{NMR}$ $\left(\mathrm{CDCl}_{3}\right): 7.30-7.42(\mathrm{~m}, 7 \mathrm{H}), 7.50-7.60(\mathrm{~m}, 6 \mathrm{H}), 8.45(\mathrm{~d}, 1 \mathrm{H}, J=7.9 \mathrm{~Hz}), 9.47(\mathrm{~s}, 1 \mathrm{H}), 9.78(\mathrm{br} \mathrm{s}, 1 \mathrm{H})$. ${ }^{13} \mathrm{C}-\mathrm{NMR}\left(\mathrm{CDCl}_{3}\right): 111.9(\mathrm{CH}), 120.6(\mathrm{CH}), 121.3(\mathrm{CH}), 123.1(\mathrm{C}), 126.7(\mathrm{C}), 127.2(\mathrm{CH}), 128.4(2 \mathrm{CH})$, $128.5(2 \mathrm{CH}), 129.2(\mathrm{CH}), 129.6(\mathrm{CH}), 130.0(2 \mathrm{CH}), 130.1(2 \mathrm{CH}), 132.4(\mathrm{C}), 134.9(\mathrm{C}), 138.4(\mathrm{C}), 138.6$ (C), $138.8(\mathrm{C}), 139.5(\mathrm{C}), 146.5(\mathrm{CH}), 153.6(\mathrm{C}), 155.9$ (C). Crystal data for $3 \mathrm{~h} . \mathrm{C}_{25} \mathrm{H}_{16} \mathrm{~N}_{4}, M=372.42$, $T=150(2) \mathrm{K}$, orthorhombic, $P b c a, a=7.1524(9), b=16.3313(17), c=33.798(4) \AA, V=3947.9(8) \AA^{3}, Z=8$, $d=1.253 \mathrm{~g} \mathrm{~cm}^{-3}, \mu=0.076 \mathrm{~mm}^{-1}$. A final refinement on $F^{2}$ with 4429 unique intensities and 265 parameters converged at $\omega R\left(F^{2}\right)=0.1564(R(F)=0.0739)$ for 3511 observed reflections with $I>2 \sigma(I)$. CCDC 1858477. This compound was also obtained in $64 \%$ yield under microwave irradiation ( $300 \mathrm{~W}$; Monowave 300, Anton Paar, Graz, Austria) for $30 \mathrm{~min}$ at $150{ }^{\circ} \mathrm{C}$.

\subsection{8-(2-Azidophenyl)-2,3-diphenylpyrido[2,3-b]pyrazine}

To a stirred solution of 8-(2-aminophenyl)-2,3-diphenylpyrido[2,3-b]pyrazine (2e, $94 \mathrm{mg}$, $0.25 \mathrm{mmol})$ in acetic acid $(1.5 \mathrm{~mL})$ at $0{ }^{\circ} \mathrm{C}$ was added $1 \mathrm{M}$ aqueous $\mathrm{NaNO}_{2}(0.35 \mathrm{~mL}, 0.35 \mathrm{mmol})$. After stirring for $1 \mathrm{~h}$ at rt, the solution was cooled to $0{ }^{\circ} \mathrm{C}$ before addition of $1 \mathrm{M}$ aqueous $\mathrm{NaN}_{3}$ $(0.35 \mathrm{~mL}, 0.35 \mathrm{mmol})$. After stirring overnight at $\mathrm{rt}, 3 \mathrm{~mL}$ of saturated aqueous $\mathrm{NaHCO}_{3}$ were added. Extraction with EtOAc $(3 \times 10 \mathrm{~mL})$, washing of the combined organic layers with brine $(10 \mathrm{~mL})$, 
drying over $\mathrm{MgSO}_{4}$, filtration and concentration under reduced pressure afforded a brown powder which was purified by chromatography over silica gel (eluent: $\mathrm{CH}_{2} \mathrm{Cl}_{2}$-EtOAc 95:5; $\mathrm{R}_{\mathrm{f}}=0.50$ ) to afford the azide in $64 \%$ yield. IR: 685, 745, 1288, 1440, 1577, 2088, 2124, $3064 \mathrm{~cm}^{-1} .{ }^{1} \mathrm{H}-\mathrm{NMR}\left(\mathrm{CDCl}_{3}\right)$ : 7.23-7.40 (m, 8H), 7.45-7.57 (m, 4H), 7.66-7.69 (m, 3H), $9.18(\mathrm{~d}, 1 \mathrm{H}, J=4.4 \mathrm{~Hz}) .{ }^{13} \mathrm{C}-\mathrm{NMR}\left(\mathrm{CDCl}_{3}\right)$ : $118.8(\mathrm{CH}), 124.7(\mathrm{CH}), 126.0(\mathrm{CH}), 127.9(\mathrm{C}), 128.2(2 \mathrm{CH}), 128.2(2 \mathrm{CH}), 129.2(\mathrm{CH}), 129.5(\mathrm{CH}), 130.1$ (2CH), $130.3(2 \mathrm{CH}), 130.4(\mathrm{CH}), 132.5(\mathrm{CH}), 134.5(\mathrm{C}), 138.3(\mathrm{C}), 138.5(\mathrm{C}), 138.7(\mathrm{C}), 146.7(\mathrm{C}), 149.8(\mathrm{C})$, $153.5(\mathrm{CH}), 153.7(\mathrm{C}), 155.8(\mathrm{C})$.

\subsection{Palladium-Catalyzed N-arylation}

\subsubsection{General Procedure 4}

To a stirred mixture of the halide $(0.50 \mathrm{mmol})$ and $\mathrm{Cs}_{2} \mathrm{CO}_{3}(0.48 \mathrm{~g}, 1.5 \mathrm{mmol})$ in 2-chloroaniline (63 $\mu \mathrm{L}, 0.60 \mathrm{mmol})$ was added a solution of the catalyst prepared by stirring $\operatorname{Pd}_{2}(\mathrm{dba})_{3}(11 \mathrm{mg}, 12.5 \mu \mathrm{mol})$ and Xantphos (16 mg, $27.5 \mu \mathrm{mol})$ in degassed dioxane $(2 \mathrm{~mL})$ for $10 \mathrm{~min}$ at $\mathrm{rt}$. The resulting mixture was heated at $110{ }^{\circ} \mathrm{C}$ for $24 \mathrm{~h}$ and cooled to rt before addition of water $(5 \mathrm{~mL})$ and extraction with EtOAc $(3 \times 10 \mathrm{~mL})$. The combined organic layers were washed with brine $(10 \mathrm{~mL})$, dried over $\mathrm{MgSO}_{4}$, filtered and concentrated under reduced pressure. The crude product was purified by chromatography over silica gel (the eluent is given in the product description).

\subsubsection{5-(2-Chlorophenylamino)-2,3-diphenylquinoxaline (1f)}

The general procedure 4 using 5-iodo-2,3-diphenylquinoxaline (1b, 0.20 g) gave 1f (eluent: heptane- $\left.\mathrm{CH}_{2} \mathrm{Cl}_{2} 60: 40 ; \mathrm{R}_{\mathrm{f}}=0.42\right)$ in $92 \%$ yield as a yellow powder. Mp: $182{ }^{\circ} \mathrm{C}$. IR: $695,729,748,959$, 1021, 1055, 1072, 1098, 1182, 1218, 1317, 1343, 1356, 1394, 1442, 1454, 1497, 1534, 1562, 1579, 1594, 1613, 3060, $3347 \mathrm{~cm}^{-1} .{ }^{1} \mathrm{H}-\mathrm{NMR}\left(\mathrm{CDCl}_{3}\right): 6.95(\mathrm{td}, 1 \mathrm{H}, J=7.7$ and $1.4 \mathrm{~Hz}), 7.27-7.39(\mathrm{~m}, 7 \mathrm{H}), 7.46-7.52(\mathrm{~m}$, 2H), 7.55-7.62 (m, 4H), 7.64-7.66 (m, 2H), $7.71(\mathrm{dd}, 1 \mathrm{H}, J=8.2$ and $1.4 \mathrm{~Hz}), 8.56(\mathrm{br} \mathrm{s}, 1 \mathrm{H}) .{ }^{13} \mathrm{C}-\mathrm{NMR}$ $\left(\mathrm{CDCl}_{3}\right)$ : $109.1(\mathrm{CH}), 118.9(\mathrm{CH}), 118.9(\mathrm{CH}), 122.6(\mathrm{CH}), 125.1(\mathrm{C}), 127.6(\mathrm{CH}), 128.2(2 \mathrm{CH}), 128.4(2 \mathrm{CH})$, $128.9(\mathrm{CH}), 128.9(\mathrm{CH}), 129.9(2 \mathrm{CH}), 130.1(2 \mathrm{CH}), 130.2(\mathrm{CH}), 130.9(\mathrm{CH}), 132.1(\mathrm{C}), 138.4(\mathrm{C}), 138.9(\mathrm{C})$, 139.2 (C), 139.3 (C), 141.9 (C), 150.4 (C), 153.9 (C). Anal. Calc. for $\mathrm{C}_{26} \mathrm{H}_{18} \mathrm{ClN}_{3}$ (407.90): C 76.56, H 4.45, N, 10.30. Found: C 76.89, H 4.58, N, 10.13.

\subsubsection{8-(2-Chlorophenylamino)-2,3-diphenylpyrido[2,3-b]pyrazine (2f)}

The general procedure 4 using 8-iodo-2,3-diphenylpyrido[2,3-b]pyrazine (2b-I, 0.20 g) gave $\mathbf{2 f}$ (eluent: $\mathrm{CH}_{2} \mathrm{Cl}_{2}$-EtOAc 90:10; $\mathrm{R}_{\mathrm{f}}=0.32$ ) in $67 \%$ yield as a yellow powder. Mp: $202{ }^{\circ} \mathrm{C}$. IR: $542,699,755$, 1021, 1102, 1242, 1313, 1336, 1356, 1437, 1452, 1534, 1558, 1583, 1646, 3060, 3322, $3631 \mathrm{~cm}^{-1} .{ }^{1} \mathrm{H}-\mathrm{NMR}$ $\left(\mathrm{CDCl}_{3}\right): 7.13-7.19(\mathrm{~m}, 2 \mathrm{H}), 7.30-7.41(\mathrm{~m}, 7 \mathrm{H}), 7.53(\mathrm{dd}, 1 \mathrm{H}, J=8.0$ and $1.5 \mathrm{~Hz}), 7.57-7.65(\mathrm{~m}, 4 \mathrm{H})$, $7.68(\mathrm{dd}, 1 \mathrm{H}, J=8.1$ and $1.5 \mathrm{~Hz}), 8.77(\mathrm{br} \mathrm{s}, 1 \mathrm{H}, \mathrm{NH}), 8.81\left(\mathrm{~d}, 1 \mathrm{H}, J=5.4 \mathrm{~Hz}, \mathrm{H}_{6}\right) .{ }^{13} \mathrm{C}-\mathrm{NMR}\left(\mathrm{CDCl}_{3}\right)$ : $102.8(\mathrm{CH}), 122.2(\mathrm{CH}), 125.5(\mathrm{CH}), 127.2(\mathrm{C}), 127.5(\mathrm{C}), 127.8(\mathrm{CH}), 128.2(2 \mathrm{CH}), 128.4(2 \mathrm{CH}), 129.2(\mathrm{CH})$, $129.4(\mathrm{CH}), 130.0(2 \mathrm{CH}), 130.3(2 \mathrm{CH}), 130.5(\mathrm{CH}), 136.2(\mathrm{C}), 138.4(\mathrm{C}), 138.5(\mathrm{C}), 147.0(\mathrm{C}), 150.3(\mathrm{C})$, $151.1(\mathrm{C}), 155.0(\mathrm{CH}), 156.5(\mathrm{C})$. Crystal data for $2 \mathrm{f} . \mathrm{C}_{25} \mathrm{H}_{17} \mathrm{ClN}_{4}, M=408.88, \mathrm{~T}=150(2) \mathrm{K}$, orthorhombic, $P$ c $a$ 2 $1, a=15.3485(15), b=18.8937(16), c=6.9936(7) \AA, V=2028.1(3) \AA^{3}, Z=4, d=1.339 \mathrm{~g} \mathrm{~cm}^{-3}$, $\mu=0.208 \mathrm{~mm}^{-1}$. A final refinement on $F^{2}$ with 4578 unique intensities and 274 parameters converged at $\omega R\left(F^{2}\right)=0.1478(R(F)=0.0583)$ for 4133 observed reflections with $I>2 \sigma(I)$. CCDC 1858474 .

\subsection{Palladium-Catalyzed N-arylation}

2,3-Diphenyl-11H-pyrazino[2,3-a]carbazole (1g) was prepared by adapting a reported procedure [40]. To a stirred mixture of 5-(2-chlorophenylamino)-2,3-diphenylquinoxaline (1f, $0.24 \mathrm{~g}$, $0.60 \mathrm{mmol})$ and 1,8-diazabicyclo[5.4.0]undec-7-ene $(0.13 \mathrm{~mL}, 0.90 \mathrm{mmol})$, was added a solution of the catalyst prepared by stirring $\mathrm{Pd}_{2}(\mathrm{dba})_{3}(14 \mathrm{mg}, 15 \mu \mathrm{mol})$ and $\mathrm{P}(t \mathrm{Bu})_{3}(12 \mathrm{mg}, 60 \mu \mathrm{mol})$ in degassed dioxane $(1 \mathrm{~mL})$ for $10 \mathrm{~min}$ at $\mathrm{rt}$. The resulting mixture was heated by microwave irradiation (300 W; Monowave 300, Anton Paar, Graz, Austria) for $10 \mathrm{~min}$ at $180^{\circ} \mathrm{C}$ before addition of water $(5 \mathrm{~mL})$ 
and extraction with EtOAc $(3 \times 10 \mathrm{~mL})$. The combined organic layers were washed with brine $(10$ $\mathrm{mL})$, dried over $\mathrm{MgSO}_{4}$, filtered and concentrated under reduced pressure. The crude product was purified by chromatography over silica gel (eluent: heptane- $\mathrm{CH}_{2} \mathrm{Cl}_{2} 60: 40 ; \mathrm{R}_{\mathrm{f}}=0.48$ ) to give $1 \mathrm{~g}$ in $62 \%$ yield as a yellow powder. Mp: $26{ }^{\circ} \mathrm{C}$. IR: 1025, 1087, 1102, 1175, 1242, 1326, 1347, 1362, 1384, 1444, $1459,1624,1731,2854,2922,3420 \mathrm{~cm}^{-1} .{ }^{1} \mathrm{H}-\mathrm{NMR}\left(\left(\mathrm{CD}_{3}\right)_{2} \mathrm{SO}\right): 6.86(\mathrm{ddd}, 1 \mathrm{H}, J=8.0,7.1$ and $1.0 \mathrm{~Hz})$, 6.91-6.97 (m, 6H), 7.01-7.10 (m, 3H), 7.14-7.17 (m, 2H), $7.28(\mathrm{~d}, 1 \mathrm{H}, J=8.3 \mathrm{~Hz}), 7.39(\mathrm{~d}, 1 \mathrm{H}, J=8.7 \mathrm{~Hz})$, $7.84(\mathrm{~d}, 1 \mathrm{H}, J=7.8 \mathrm{~Hz}), 8.14(\mathrm{~d}, 1 \mathrm{H}, J=8.7 \mathrm{~Hz}), 12.13(\mathrm{br} \mathrm{s}, 1 \mathrm{H}) .{ }^{13} \mathrm{C}-\mathrm{NMR}\left(\left(\mathrm{CD}_{3}\right)_{2} \mathrm{SO}\right): 112.2(\mathrm{CH}), 118.8$ $(\mathrm{CH}), 119.7(\mathrm{CH}), 120.3(\mathrm{CH}), 120.7(\mathrm{C}), 122.7(\mathrm{C}), 124.2(\mathrm{CH}), 125.6(\mathrm{CH}), 128.0(2 \mathrm{CH}), 128.0(2 \mathrm{CH})$, $128.5(\mathrm{CH}), 128.6(\mathrm{CH}), 129.7(2 \mathrm{CH}), 129.9(2 \mathrm{CH}), 130.0(\mathrm{C}), 134.3(\mathrm{C}), 139.0(\mathrm{C}), 139.2(\mathrm{C}), 139.8(\mathrm{C})$, 139.9 (C), 150.7 (C), 151.4 (C). Anal. Calc. for $\mathrm{C}_{26} \mathrm{H}_{17} \mathrm{~N}_{3}$ (371.44): C 84.07, H 4.61, N, 11.31. Found: C 84.19, H 4.52, N, 11.12 .

\subsection{One-Pot Palladium-Catalyzed N-arylation/C-H Arylation}

\subsubsection{General Procedure 5}

To a mixture of the halide ( $0.25 \mathrm{mmol}), 1,8$-diazabicyclo[5.4.0]undec-7-ene (118 $\mu \mathrm{L}, 0.75 \mathrm{mmol})$, 2-chloroaniline ( $38 \mathrm{mg}, 0.30 \mathrm{mmol}), \mathrm{Pd}_{2}(\mathrm{dba})_{3}(9.2 \mathrm{mg}, 10 \mu \mathrm{mol})$ and Xantphos (13 mg, $\left.22 \mu \mathrm{mol}\right)$, was added degassed 1,4-dioxane (1 mL). The mixture was heated by microwave irradiation (150 W; Monowave 300, Anton Paar, Graz, Austria) under the conditions given in the product description. The cooled residue was taken up with EtOAc $(20 \mathrm{~mL})$. The organic layer was washed with brine $(10 \mathrm{~mL})$, dried over $\mathrm{MgSO}_{4}$, filtered and concentrated under reduced pressure. The crude product was purified by chromatography over silica gel (the eluent is given in the product description).

\subsubsection{2,3-Diphenyl-11H-pyrazino[ $\left[2^{\prime}, 3^{\prime}: 5,6\right]$ pyrido[4,3-b]indole (2g)}

The general procedure $5\left(1 \mathrm{~h}\right.$ at $\left.180^{\circ} \mathrm{C}\right)$ using 8-iodo-2,3-diphenylpyrido[2,3-b]pyrazine (2b-I, $0.10 \mathrm{~g}$ ) gave $2 \mathrm{~g}$ (eluent: $\mathrm{CH}_{2} \mathrm{Cl}_{2}$-EtOAc 90:10; $\mathrm{R}_{\mathrm{f}}=0.43$ ) in $70 \%$ yield as a white powder. $\mathrm{Mp}>260{ }^{\circ} \mathrm{C}$. IR: 525, 542, 551, 626, 699, 750, 768, 1025, 1045, 1075, 1100, 1236, 1339, 1373, 1444, 1555, 1736, 2665, $3056 \mathrm{~cm}^{-1} .{ }^{1} \mathrm{H}-\mathrm{NMR}\left(\left(\mathrm{CD}_{3}\right)_{2} \mathrm{SO}\right): 7.40-7.45(\mathrm{~m}, 7 \mathrm{H}), 7.55-7.63(\mathrm{~m}, 5 \mathrm{H}), 7.77(\mathrm{dd}, 1 \mathrm{H}, J=8.2$ and $0.9 \mathrm{~Hz})$, $8.42(\mathrm{dt}, 1 \mathrm{H}, J=7.8$ and $1.0 \mathrm{~Hz}), 9.87(\mathrm{~s}, 1 \mathrm{H}), 13.19(\mathrm{~s}, 1 \mathrm{H}) .{ }^{13} \mathrm{C}-\mathrm{NMR}\left(\left(\mathrm{CD}_{3}\right)_{2} \mathrm{SO}\right): 112.6(\mathrm{CH}), 118.2(\mathrm{C})$, $120.7(\mathrm{CH}), 121.3(\mathrm{CH}), 121.4(\mathrm{C}), 126.4(\mathrm{C}), 126.5(\mathrm{CH}), 128.0(\mathrm{CH}), 128.0(2 \mathrm{CH}), 128.1(2 \mathrm{CH}), 128.8$ $(\mathrm{CH}), 129.8(2 \mathrm{CH}), 129.9(2 \mathrm{CH}), 138.6(\mathrm{C}), 138.7(\mathrm{C}), 139.4(\mathrm{C}), 140.0(\mathrm{C}), 147.4(\mathrm{C}), 148.5(\mathrm{CH}), 151.4(\mathrm{C})$, 153.4 (C). Anal. Calc. for $\mathrm{C}_{25} \mathrm{H}_{16} \mathrm{~N}_{4}$ (372.43): C 80.63, H 4.33, N, 15.04. Found: C 80.54, H 4.28, N, 14.89 .

\subsubsection{7-(Phenylamino)-2,3-diphenylpyrido[3,4-b]pyrazine (3 $\left.\mathbf{g}^{\prime}\right)$}

The general procedure $5\left(40 \mathrm{~min}\right.$ at $\left.180{ }^{\circ} \mathrm{C}\right)$ using 8-bromo-7-iodo-2,3-diphenylpyrido[3,4- $b$ ] pyrazine ( $3 \mathbf{b}, 0.12 \mathrm{~g}$ ) gave $3 \mathbf{g}^{\prime}$ (eluent: $\mathrm{CH}_{2} \mathrm{Cl}_{2}-\mathrm{MeOH} 99: 1 ; \mathrm{R}_{\mathrm{f}}=0.27$ ) in $32 \%$ yield as a yellow powder. Mp: 224-226 ${ }^{\circ} \mathrm{C}$. IR: 699, 750, 770, 978, 1025, 1057, 1077, 1169, 1197, 1261, 1336, 1349, 1435, 1450, 1527, 1555, 1588, 1613, 2854, 2927, 2961, 3025, $3232 \mathrm{~cm}^{-1} .{ }^{1} \mathrm{H}-\mathrm{NMR}\left(\mathrm{CDCl}_{3}\right): 7.14(\mathrm{p}, 1 \mathrm{H}, \mathrm{J}=4.4 \mathrm{~Hz}), 7.23$ (br s, 1H), 7.29-7.49 (m, 15H), $9.26(\mathrm{~s}, 1 \mathrm{H}) .{ }^{13} \mathrm{C}-\mathrm{NMR}\left(\mathrm{CDCl}_{3}\right)$ : $158.3(\mathrm{C}), 155.8(\mathrm{C}), 154.0(\mathrm{CH}), 151.4(\mathrm{C})$, $146.3(\mathrm{C}), 139.8(\mathrm{C}), 138.8(\mathrm{C}), 138.7(\mathrm{C}), 132.4(\mathrm{C}), 129.8(2 \mathrm{CH}), 129.7(2 \mathrm{CH}), 129.7(2 \mathrm{CH}), 129.5(\mathrm{CH})$, $128.9(\mathrm{CH}), 128.4(2 \mathrm{CH}), 128.4(2 \mathrm{CH}), 124.1(\mathrm{CH}), 121.4(2 \mathrm{CH}), 98.3(\mathrm{CH})$. Anal. Calc. for $\mathrm{C}_{25} \mathrm{H}_{18} \mathrm{~N}_{4}$ (374.45): C 80.19, H 4.85, N, 14.96. Found: C 80.17, H 4.99, N, 14.84.

\subsection{Copper-Catalyzed N-arylation}

\subsubsection{General Procedure 6}

A mixture containing the iodide $(0.50 \mathrm{mmol})$ and azole $(1.0 \mathrm{mmol}), \mathrm{Cu}_{2} \mathrm{O}(6.0 \mathrm{mg}, 0.10 \mathrm{mmol})$, $\mathrm{Cs}_{2} \mathrm{CO}_{3}(0.33 \mathrm{~g}, 1.0 \mathrm{mmol})$ and DMSO $(0.5 \mathrm{~mL})$ was stirred at $110{ }^{\circ} \mathrm{C}$ for $24 \mathrm{~h}$. The cooled residue was taken up with EtOAc $(20 \mathrm{~mL})$ and filtered through a Celite pad. The organic layer was washed with water $(10 \mathrm{~mL})$ and brine $(10 \mathrm{~mL})$, dried over $\mathrm{MgSO}_{4}$, filtered and concentrated under reduced pressure. 
The crude product was purified by chromatography over silica gel (the eluent is given in the product description).

\subsubsection{2,3-Diphenyl-8-(N-pyrrolyl)pyrido[2,3-b]pyrazine (2i)}

The general procedure 6 using 8-iodo-2,3-diphenylpyrido[2,3-b]pyrazine (2b-I, $0.20 \mathrm{~g}$ ) and pyrrole $\left(67 \mathrm{mg}\right.$ ) gave 2i (eluent: $\mathrm{CH}_{2} \mathrm{Cl}_{2}$-EtOAc 90:10; $\mathrm{R}_{\mathrm{f}}=0.47$ ) in $67 \%$ yield as a yellow powder. Mp: $210{ }^{\circ} \mathrm{C}$. IR: 946, 1025, 1072, 1096, 1107, 1173, 1238, 1289, 1328, 1362, 1388, 1433, 1454, 1482, 1549, 1588, 3025, 3060, 3111, 3141, $3180 \mathrm{~cm}^{-1}$. ${ }^{1} \mathrm{H}-\mathrm{NMR}\left(\mathrm{CDCl}_{3}\right): 6.39-6.40(\mathrm{~m}, 2 \mathrm{H}), 7.24-7.35(\mathrm{~m}, 6 \mathrm{H}), 7.49-7.53(\mathrm{~m}$, $3 \mathrm{H}), 7.59-7.62(\mathrm{~m}, 2 \mathrm{H}), 7.65-7.66(\mathrm{~m}, 2 \mathrm{H}), 9.01(\mathrm{~d}, 1 \mathrm{H}, J=5.0 \mathrm{~Hz}) .{ }^{13} \mathrm{C}-\mathrm{NMR}\left(\mathrm{CDCl}_{3}\right): 112.0(2 \mathrm{CH})$, $115.1(\mathrm{CH}), 122.7(2 \mathrm{CH}), 128.3(2 \mathrm{CH}), 128.4(2 \mathrm{CH}), 129.4(\mathrm{C}), 129.5(\mathrm{CH}), 129.8(\mathrm{CH}), 130.0(2 \mathrm{CH})$, $130.2(2 \mathrm{CH}), 137.8(\mathrm{C}), 138.1(\mathrm{C}), 144.6(\mathrm{C}), 150.7(\mathrm{C}), 153.2(\mathrm{C}), 153.9(\mathrm{CH}), 156.0(\mathrm{C})$. Crystal data for 2 i. $\mathrm{C}_{23} \mathrm{H}_{16} \mathrm{~N}_{4}, M=348.40, T=150(2) \mathrm{K}$, orthorhombic, $P 22_{1} 2_{1} 2_{1}, a=6.3672(5), b=13.0997(10)$, $c=21.5377(18) \AA, V=1796.4(2) \AA^{3}, Z=4, d=1.288 \mathrm{~g} \mathrm{~cm}^{-3}, \mu=0.079 \mathrm{~mm}^{-1}$. A final refinement on $F^{2}$ with 2367 unique intensities and 245 parameters converged at $\omega R\left(F^{2}\right)=0.1207(R(F)=0.0498)$ for 1679 observed reflections with $I>2 \sigma(I)$. CCDC 1858475 .

\subsubsection{8-(N-indolyl)-2,3-diphenylpyrido[2,3-b]pyrazine (2j)}

The general procedure 6 using 8-iodo-2,3-diphenylpyrido[2,3-b]pyrazine (2b-I, $0.20 \mathrm{~g})$ and indole ( $0.12 \mathrm{~g}$ ) gave $2 \mathrm{j}$ (eluent: $\mathrm{CH}_{2} \mathrm{Cl}_{2} ; \mathrm{R}_{\mathrm{f}}=0.36$ ) in $51 \%$ yield as a red powder. Mp: $136{ }^{\circ} \mathrm{C}$. IR: 1023,1154 , $1208,1236,1324,1356,1379,1442,1454,1478,1519,1555,1577,1592,3240,3339,3639 \mathrm{~cm}^{-1}$. ${ }^{1} \mathrm{H}-\mathrm{NMR}$ $\left(\mathrm{CDCl}_{3}\right): 6.82(\mathrm{~d}, 1 \mathrm{H}, J=3.4 \mathrm{~Hz}), 7.22-7.44(\mathrm{~m}, 8 \mathrm{H}), 7.53-7.56(\mathrm{~m}, 2 \mathrm{H}), 7.67(\mathrm{~d}, 1 \mathrm{H}, J=8.3 \mathrm{~Hz}), 7.70-7.72$ $(\mathrm{m}, 3 \mathrm{H}), 7.86(\mathrm{dd}, 1 \mathrm{H}, J=4.9$ and $1.2 \mathrm{~Hz}), 7.94(\mathrm{~d}, 1 \mathrm{H}, J=3.4 \mathrm{~Hz}), 9.17(\mathrm{~d}, 1 \mathrm{H}, J=4.9 \mathrm{~Hz}) .{ }^{13} \mathrm{C}-\mathrm{NMR}$ $\left(\mathrm{CDCl}_{3}\right)$ : $106.1(\mathrm{CH}), 111.4(\mathrm{CH}), 118.0(\mathrm{CH}), 121.5(\mathrm{CH}), 122.1(\mathrm{CH}), 123.2(\mathrm{CH}), 128.4(2 \mathrm{CH}), 128.4$ $(2 \mathrm{CH}), 128.5(\mathrm{C}), 129.7(\mathrm{CH}), 129.9(\mathrm{CH}), 130.1(2 \mathrm{CH}), 130.3(\mathrm{C}), 130.3(2 \mathrm{CH}), 130.5(\mathrm{C}), 130.8(\mathrm{CH}), 136.2$ (C), 137.8 (C), 138.0 (C), 144.8 (C), $150.8(\mathrm{C}), 153.6(\mathrm{CH}), 156.4$ (C). Anal. Calc. for $\mathrm{C}_{27} \mathrm{H}_{18} \mathrm{~N}_{4}$ (398.47): C 81.39, H 4.55, N, 14.06. Found: C 81.26, H 4.67, N, 13.84 .

\subsubsection{2,3-Diphenyl-8-(N-pyrazolyl)pyrido[2,3-b]pyrazine (2k)}

The general procedure 6 using 8-iodo-2,3-diphenylpyrido[2,3-b]pyrazine (2b-I, $0.20 \mathrm{~g})$ and pyrazole $\left(68 \mathrm{mg}\right.$ ) gave $\mathbf{2 k}$ (eluent: $\mathrm{CH}_{2} \mathrm{Cl}_{2}$-EtOAc 80:20; $\left.\mathrm{R}_{\mathrm{f}}=0.47\right)$ in $71 \%$ yield as a pale yellow powder. Mp: $200{ }^{\circ} \mathrm{C}$. IR: 1027, 1032, 1092, 1164, 1229, 1324, 1356, 1388, 1532, 1549, 1592, 3034, 3060 , $3159 \mathrm{~cm}^{-1} .{ }^{1} \mathrm{H}-\mathrm{NMR}\left(\mathrm{CDCl}_{3}\right): 6.55(\mathrm{~d}, 1 \mathrm{H}, J=2.2 \mathrm{~Hz}), 7.30-7.41(\mathrm{~m}, 6 \mathrm{H}), 7.55-7.58(\mathrm{~m}, 2 \mathrm{H}), 7.64-7.66$ $(\mathrm{m}, 2 \mathrm{H}), 7.82(\mathrm{~s}, 1 \mathrm{H}), 8.34(\mathrm{dd}, 1 \mathrm{H}, J=5.3$ and $2.4 \mathrm{~Hz}), 9.12(\mathrm{dd}, 1 \mathrm{H}, J=5.2$ and $2.2 \mathrm{~Hz}), 9.46(\mathrm{t}, 1 \mathrm{H}$, $J=2.5 \mathrm{~Hz}) .{ }^{13} \mathrm{C}-\mathrm{NMR}\left(\mathrm{CDCl}_{3}\right): 109.2(\mathrm{CH}), 115.0(\mathrm{CH}), 127.9(\mathrm{C}), 128.3(2 \mathrm{CH}), 128.6(2 \mathrm{CH}), 129.6(\mathrm{CH})$, $129.8(\mathrm{CH}), 129.9(2 \mathrm{CH}), 130.3(2 \mathrm{CH}), 134.4(\mathrm{CH}), 137.6(\mathrm{C}), 138.3(\mathrm{C}), 142.5(\mathrm{CH}), 143.2(\mathrm{C}), 150.5(\mathrm{C})$, 153.4 (C), $154.3(\mathrm{CH}), 156.1$ (C). Anal. Calc. for $\mathrm{C}_{22} \mathrm{H}_{15} \mathrm{~N}_{5}$ (349.40): C 75.63, H 4.33, N, 20.04. Found: C 75.71, H 4.42, N, 19.86 .

\subsubsection{8-(N-imidazolyl)-2,3-diphenylpyrido[2,3-b]pyrazine (21)}

The general procedure 6 using 8-iodo-2,3-diphenylpyrido[2,3-b]pyrazine (2b-I, $0.20 \mathrm{~g})$ and imidazole (68 mg) gave 21 (eluent: EtOAc-MeOH 95:5; $\mathrm{R}_{\mathrm{f}}=0.48$ ) in $69 \%$ yield as a yellow powder. Mp: $209^{\circ} \mathrm{C}$. IR: 1019, 1053, 1075, 1105, 1115, 1169, 1236, 1319, 1334, 1379, 1429, 1446, 1459, 1482, 1549 , 1594, 3064, 3124, $3639 \mathrm{~cm}^{-1} .{ }^{1} \mathrm{H}-\mathrm{NMR}\left(\mathrm{CDCl}_{3}\right): 7.32-7.45(\mathrm{~m}, 7 \mathrm{H}), 7.56(\mathrm{~d}, 2 \mathrm{H}, J=6.6 \mathrm{~Hz}), 7.65-7.71(\mathrm{~m}$, 3H), $7.80(\mathrm{br} \mathrm{s}, 1 \mathrm{H}), 8.82(\mathrm{br} \mathrm{s}, 1 \mathrm{H}), 9.20(\mathrm{~d}, 1 \mathrm{H}, J=4.8 \mathrm{~Hz}) .{ }^{13} \mathrm{C}-\mathrm{NMR}\left(\mathrm{CDCl}_{3}\right): 115.6(\mathrm{CH}), 119.5(\mathrm{CH})$, $128.3(2 \mathrm{CH}), 128.4(2 \mathrm{CH}), 128.9(\mathrm{C}), 129.7(\mathrm{CH}), 129.9(\mathrm{CH}), 129.9(2 \mathrm{CH}), 130.1(2 \mathrm{CH}), 130.3(\mathrm{CH})$, $137.5(\mathrm{C}), 137.6(\mathrm{C}), 138.8(\mathrm{CH}), 141.4(\mathrm{C}), 150.7(\mathrm{C}), 154.1(\mathrm{C}), 154.2(\mathrm{CH}), 156.6(\mathrm{C})$. Anal. Calc. for $\mathrm{C}_{22} \mathrm{H}_{15} \mathrm{~N}_{5}$ (349.40): C 75.63, H 4.33, N, 20.04. Found: C 75.74, H 4.37, N, 19.92. 


\subsubsection{2,3-Diphenyl-8-[1-(1,2,4-triazolyl)]pyrido[2,3-b]pyrazine (2m)}

The general procedure 6 using 8-iodo-2,3-diphenylpyrido[2,3-b]pyrazine (2b-I, $0.20 \mathrm{~g})$ and 1,2,4-triazole (69 mg) gave $2 \mathrm{~m}$ (eluent: $\mathrm{CH}_{2} \mathrm{Cl}_{2}$-EtOAc 80:20; $\mathrm{R}_{\mathrm{f}}=0.35$ ) in $79 \%$ yield as an orange powder. Mp: $205^{\circ} \mathrm{C}$. IR: 708, 995, 1025, 1049, 1079, 1124, 1158, 1223, 1242, 1276, 1332, 1386, 1403, 1459, 1508, 1551, 1590, 3064, $3146 \mathrm{~cm}^{-1} .{ }^{1} \mathrm{H}-\mathrm{NMR}\left(\mathrm{CDCl}_{3}\right): 7.35-7.49(\mathrm{~m}, 6 \mathrm{H}), 7.59(\mathrm{~d}, 2 \mathrm{H}, J=7.0 \mathrm{~Hz})$, $7.68(\mathrm{~d}, 2 \mathrm{H}, J=7.2 \mathrm{~Hz}), 8.22(\mathrm{~s}, 1 \mathrm{H}), 8.37$ (br s, $1 \mathrm{H}), 9.27$ (br s, $1 \mathrm{H}), 10.16$ (br s, $1 \mathrm{H}) .{ }^{13} \mathrm{C}-\mathrm{NMR}\left(\mathrm{CDCl}_{3}\right)$ : $115.1(\mathrm{CH}), 127.3(\mathrm{C}), 128.2(2 \mathrm{CH}), 128.5(2 \mathrm{CH}), 129.8(\mathrm{CH}), 129.8(2 \mathrm{CH}), 129.9(\mathrm{CH}), 130.1(2 \mathrm{CH})$, 137.3 (C), $137.7(\mathrm{C}), 140.4(\mathrm{C}), 147.2(\mathrm{CH}), 150.4(\mathrm{C}), 152.0(\mathrm{CH}), 154.1(\mathrm{C}), 154.5(\mathrm{CH}), 156.7(\mathrm{C})$. Anal. Calc. for $\mathrm{C}_{21} \mathrm{H}_{14} \mathrm{~N}_{6}$ (350.39): C 71.99, H 4.03, N, 23.99. Found: C 72.19, H 4.15, N, 23.81.

\subsubsection{5-Iodo-2,3-diphenyl-8-(N-pyrazolyl)quinoxaline ( $\left.\mathbf{1 k}^{\prime}\right)$}

The general procedure 6 using 5-iodo-2,3-diphenylquinoxaline $\left(\mathbf{1 b}^{\prime}, 0.27 \mathrm{~g}\right)$ and pyrazole $(68 \mathrm{mg})$ gave $1 \mathbf{k}^{\prime}$ (eluent: $\mathrm{CH}_{2} \mathrm{Cl}_{2}$-heptane 80:20; $\mathrm{R}_{\mathrm{f}}=0.45$ ) in $50 \%$ yield as an pale yellow powder. Mp: 200-202 ${ }^{\circ}$ C. IR: 536, 585, 602, 692, 696, 755, 843, 894, 946, 1040, 1092, 1182, 1193, 1221, 1336, 1397, 1465, 1519, 1543, 1592, 3060, $3159 \mathrm{~cm}^{-1} .{ }^{1} \mathrm{H}-\mathrm{NMR}\left(\mathrm{CDCl}_{3}\right): 6.56(\mathrm{dd}, 1 \mathrm{H}, J=2.6,1.8 \mathrm{~Hz}), 7.35-7.45(\mathrm{~m}, 6 \mathrm{H}), 7.57-7.60$ $(\mathrm{m}, 2 \mathrm{H}), 7.70-7.73(\mathrm{~m}, 2 \mathrm{H}), 7.82(\mathrm{~d}, 1 \mathrm{H}, J=1.8 \mathrm{~Hz}), 8.12(\mathrm{~d}, 1 \mathrm{H}, J=8.2 \mathrm{~Hz}), 8.43(\mathrm{~d}, 1 \mathrm{H}, J=8.3 \mathrm{~Hz})$, 8.97-8.98 (m, 1H). ${ }^{13} \mathrm{C}-\mathrm{NMR}\left(\mathrm{CDCl}_{3}\right): 99.3(\mathrm{C}), 107.6(\mathrm{CH}), 123.7(\mathrm{CH}), 128.4(2 \mathrm{CH}), 128.5(2 \mathrm{CH}), 129.5$ (CH), $129.6(\mathrm{CH}), 130.0(2 \mathrm{CH}), 130.4(2 \mathrm{CH}), 133.2(\mathrm{C}), 133.5(\mathrm{CH}), 137.1(\mathrm{C}), 137.8(\mathrm{C}), 138.2(\mathrm{C}), 139.7$ $(\mathrm{CH}), 140.7(\mathrm{C}), 141.1(\mathrm{CH}), 153.0(\mathrm{C}), 153.6(\mathrm{C})$. Anal. Calc. for $\mathrm{C}_{23} \mathrm{H}_{15} \mathrm{IN}_{4}$ (474.31): C 58.24, H 3.19, N, 11.81. Found: C 58.33, H 3.26, N, 11.68.

\subsection{Nucleophilic Substitution Using Amines}

\subsubsection{General Procedure 7}

A sealed tube containing the iodide $(0.50 \mathrm{mmol})$ and amine (amount given in the product description) in ethanol $(2 \mathrm{~mL})$ was heated (conditions given in the product description). The cooled residue was concentrated before chromatography over silica gel (eluent given in the product description).

\subsubsection{8-(Isopropylamino)-2,3-diphenylpyrido[2,3-b]pyrazine (2n)}

The general procedure $7\left(150{ }^{\circ} \mathrm{C}, 18 \mathrm{~h}\right)$ using 8-iodo-2,3-diphenylpyrido[2,3-b]pyrazine (2b-I, 0.20 $\mathrm{g}$ ) and isopropylamine $\left(51 \mu \mathrm{L}, 0.60 \mathrm{mmol}\right.$ ) gave $2 \mathrm{n}$ (eluent: $\mathrm{CH}_{2} \mathrm{Cl}_{2}$-EtOAc 50:50; $\left.\mathrm{R}_{\mathrm{f}}=0.20\right)$ in $69 \%$ yield as a beige powder. Mp: $179^{\circ} \mathrm{C}$. IR: $699,703,772,804,1156,1178,1236,1313,1336,1538,1564,1592$, 2965, 3038, 3064, $3390 \mathrm{~cm}^{-1} .{ }^{1} \mathrm{H}-\mathrm{NMR}\left(\mathrm{CDCl}_{3}\right): 1.27(\mathrm{~d}, 6 \mathrm{H}, J=6.4 \mathrm{~Hz}, \mathrm{Me}), 3.77(\mathrm{dp}, 1 \mathrm{H}, J=7.9$ and $\left.6.4 \mathrm{~Hz}, \mathrm{CHMe}_{2}\right), 6.40(\mathrm{br} \mathrm{d}, 1 \mathrm{H}, J=8.0 \mathrm{~Hz}, \mathrm{NH}), 6.45(\mathrm{~d}, 1 \mathrm{H}, J=5.5 \mathrm{~Hz}), 7.15-7.28(\mathrm{~m}, 6 \mathrm{H}), 7.39-7.42$ $(\mathrm{m}, 2 \mathrm{H}), 7.46-7.49(\mathrm{~m}, 2 \mathrm{H}), 8.59(\mathrm{dd}, 1 \mathrm{H}, J=5.4,0.6 \mathrm{~Hz}) .{ }^{13} \mathrm{C}-\mathrm{NMR}\left(\mathrm{CDCl}_{3}\right): 22.3\left(2 \mathrm{CH}_{3}\right), 44.1(\mathrm{CH})$, $100.7(\mathrm{CH}), 127.1(\mathrm{C}), 128.0(2 \mathrm{CH}), 128.2(2 \mathrm{CH}), 128.7(\mathrm{CH}), 129.0(\mathrm{CH}), 129.9(2 \mathrm{CH}), 130.2(2 \mathrm{CH}), 138.4$ (C), 138.9 (C), 149.8 (C), 150.1 (C), 150.1 (C), $154.8(\mathrm{CH}), 155.8$ (C). Anal. Calc. for $\mathrm{C}_{22} \mathrm{H}_{20} \mathrm{~N}_{4}(340.43)$ : C 77.62, H 5.92, N, 16.46. Found: C 77.72, H 6.14, N, 16.19.

\subsubsection{8-(4-Methoxybenzylamino)-2,3-diphenylpyrido[2,3-b]pyrazine (2o)}

The general procedure $7\left(150{ }^{\circ} \mathrm{C}, 24 \mathrm{~h}\right)$ using 8-iodo-2,3-diphenylpyrido[2,3-b]pyrazine (2b-I, $0.20 \mathrm{~g})$ and 4-methoxybenzylamine $\left(78 \mu \mathrm{L}, 0.60 \mathrm{mmol}\right.$ ) gave $2 \mathrm{o}$ (eluent: $\mathrm{CH}_{2} \mathrm{Cl}_{2}$-EtOAc 50:50; $\mathrm{R}_{\mathrm{f}}=$ 0.48 ) in $71 \%$ yield as a yellow powder. Mp: $190^{\circ} \mathrm{C}$. IR: 697, 832, 1175, 1236, 1302, 1341, 1437, 1459, 1510, 1585, 2828, 2910, 3064, $3232 \mathrm{~cm}^{-1} .{ }^{1} \mathrm{H}-\mathrm{NMR}\left(\mathrm{CDCl}_{3}\right): 3.81(\mathrm{~s}, 3 \mathrm{H}, \mathrm{OMe}), 4.55(\mathrm{~d}, 2 \mathrm{H}, J=5.9 \mathrm{~Hz})$, $6.58(\mathrm{~d}, 1 \mathrm{H}, J=5.3 \mathrm{~Hz}), 6.90(\mathrm{~d}, 2 \mathrm{H}, J=8.7 \mathrm{~Hz}), 7.02(\mathrm{t}, 1 \mathrm{H}, J=5.7 \mathrm{~Hz}), 7.27-7.35(\mathrm{~m}, 8 \mathrm{H}), 7.49-7.51(\mathrm{~m}$, 2H), 7.58-7.61 (m, 2H), $8.69(\mathrm{~d}, J=5.3 \mathrm{~Hz}, 1 \mathrm{H}) .{ }^{13} \mathrm{C}-\mathrm{NMR}\left(\mathrm{CDCl}_{3}\right): 46.5\left(\mathrm{CH}_{2}\right), 55.3\left(\mathrm{CH}_{3}\right), 101.1(\mathrm{CH})$, $114.3(2 \mathrm{CH}), 127.2(\mathrm{C}), 128.0(2 \mathrm{CH}), 128.2(2 \mathrm{CH}), 128.6(2 \mathrm{CH}), 128.8(\mathrm{CH}), 129.1(\mathrm{CH}), 129.1(\mathrm{C}), 129.9$ (2CH), $130.3(2 \mathrm{CH}), 138.5(\mathrm{C}), 138.8(\mathrm{C}), 150.0(\mathrm{C}), 150.2(\mathrm{C}), 150.9(\mathrm{C}), 154.9(\mathrm{CH}), 155.9(\mathrm{C}), 159.2(\mathrm{C})$. Anal. Calc. for $\mathrm{C}_{27} \mathrm{H}_{22} \mathrm{~N}_{4} \mathrm{O}$ (418.50): C 77.49, H 5.30, N, 13.39. Found: C 77.58, H 5.44, N, 13.20. 


\subsubsection{8-(Benzylamino)-2,3-diphenylpyrido[2,3-b]pyrazine (2p)}

The general procedure $7\left(150{ }^{\circ} \mathrm{C}, 24 \mathrm{~h}\right)$ using 8-iodo-2,3-diphenylpyrido[2,3-b]pyrazine (2b-I, $0.20 \mathrm{~g})$ and benzylamine $(66 \mu \mathrm{L}, 0.60 \mathrm{mmol})$ gave $2 p$ (eluent: $\mathrm{CH}_{2} \mathrm{Cl}_{2}$-EtOAc 50:50; $\left.\mathrm{R}_{\mathrm{f}}=0.50\right)$ in $79 \%$ yield as a yellow powder. Mp: $238^{\circ} \mathrm{C}$. IR: 697, 768, 873, 1150, 1238, 1300, 1324, 1339, 1439, 1538, 1590, 2910, 3064, $3201 \mathrm{~cm}^{-1} .{ }^{1} \mathrm{H}-\mathrm{NMR}\left(\mathrm{CDCl}_{3}\right): 4.63(\mathrm{~d}, 2 \mathrm{H}, J=6.0 \mathrm{~Hz}), 6.56(\mathrm{~d}, 1 \mathrm{H}, J=5.4 \mathrm{~Hz}), 7.11(\mathrm{t}, 1 \mathrm{H}$, $J=6.0 \mathrm{~Hz}), 7.27-7.39(\mathrm{~m}, 11 \mathrm{H}), 7.49-7.52(\mathrm{~m}, 2 \mathrm{H}), 7.58-7.61(\mathrm{~m}, 2 \mathrm{H}), 8.68(\mathrm{~d}, 1 \mathrm{H}, J=5.4 \mathrm{~Hz}) .{ }^{13} \mathrm{C}-\mathrm{NMR}$ $\left(\mathrm{CDCl}_{3}\right): 47.0\left(\mathrm{CH}_{2}\right), 101.2(\mathrm{CH}), 127.2(2 \mathrm{CH}), 127.2(\mathrm{C}), 127.8(\mathrm{CH}), 128.1(2 \mathrm{CH}), 128.3(2 \mathrm{CH}), 128.9$ $(\mathrm{CH}), 128.9(2 \mathrm{CH}), 129.1(\mathrm{CH}), 129.9(2 \mathrm{CH}), 130.3(2 \mathrm{CH}), 137.2(\mathrm{C}), 138.5(\mathrm{C}), 138.8(\mathrm{C}), 150.0(\mathrm{C})$, $150.3(\mathrm{C}), 151.0(\mathrm{C}), 154.9(\mathrm{CH}), 156.0(\mathrm{C})$. Crystal data for $2 \mathbf{p} . \mathrm{C}_{26} \mathrm{H}_{20} \mathrm{~N}_{4}, M=388.46, T=150(2) \mathrm{K}$, monoclinic, $P 22_{1} / n, a=6.0721(6), b=12.8640(10), c=25.460(2) \AA, \beta=91.436(4){ }^{\circ}, V=1988.1(3) \AA^{3}$, $Z=4, d=1.298 \mathrm{~g} \mathrm{~cm}^{-3}, \mu=0.078 \mathrm{~mm}^{-1}$. A final refinement on $F^{2}$ with 4438 unique intensities and 274 parameters converged at $\omega R\left(F^{2}\right)=0.1432(R(F)=0.0626)$ for 3710 observed reflections with $I>2 \sigma(I)$. CCDC 1858476.

3.11. Nucleophilic Substitution using Hydrazine Hydrate: 8-Hydrazino-2,3-diphenylpyrido[2,3-b]pyrazine (2q)

A solution of 8-iodo-2,3-diphenyl pyrido [2,3-b]pyrazine (2b-I, $0.20 \mathrm{~g}, 0.50 \mathrm{mmol})$ and hydrazine hydrate $(0.25 \mathrm{~mL}, 5.0 \mathrm{mmol})$ in isopropanol $(2 \mathrm{~mL})$ was heated under reflux for $4 \mathrm{~h}$. The cooled residue was concentrated and taken up with EtOAc $(20 \mathrm{~mL})$. The organic layer was washed with water $(10 \mathrm{~mL})$, dried over $\mathrm{MgSO}_{4}$, filtered and concentrated under reduced pressure to give the title compound $\mathbf{2 q}$ in $92 \%$ yield as a red powder. $\mathrm{Mp}>250{ }^{\circ} \mathrm{C} .{ }^{1} \mathrm{H}-\mathrm{NMR}\left(\mathrm{CDCl}_{3}\right): 4.22(\mathrm{br} \mathrm{s}, 2 \mathrm{H}, \mathrm{NH}), 6.91(\mathrm{~d}, 1 \mathrm{H}, J=5.6 \mathrm{~Hz})$, 7.22-7.36 (m, 7H), 7.40-7.43 (m, 2H), 7.48-7.51 (m, 2H), $8.60(\mathrm{~d}, 1 \mathrm{H}, J=5.6 \mathrm{~Hz}) .{ }^{13} \mathrm{C}-\mathrm{NMR}\left(\mathrm{CDCl}_{3}\right)$ : $101.2(\mathrm{CH}), 126.2(\mathrm{C}), 128.2(2 \mathrm{CH}), 128.3(2 \mathrm{CH}), 129.0(\mathrm{CH}), 129.2(\mathrm{CH}), 129.9(2 \mathrm{CH}), 130.3(2 \mathrm{CH})$, 138.4 (C), 138.7 (C), 149.7 (C), 150.3 (C), $153.0(\mathrm{C}), 155.0(\mathrm{CH}), 156.1$ (C). Anal. Calc. for $\mathrm{C}_{19} \mathrm{H}_{15} \mathrm{~N}_{5}$ (313.36): C 72.83, H 4.83, N, 22.35. Found: C 72.96, H 4.89, N, 22.31.

\subsection{Condensation Reactions from the Hydrazine $2 q$}

\subsubsection{General Procedure 8}

A sealed tube containing 8-hydrazino-2,3-diphenylpyrido[2,3-b] pyrazine (2q, $0.16 \mathrm{~g}, 0.50 \mathrm{mmol})$ and the aldehyde $(0.55 \mathrm{mmol})$ in ethanol $(2 \mathrm{~mL})$ was heated at $110^{\circ} \mathrm{C}$ overnight. The cooled residue was concentrated under vacuum, washed with methanol and isolated by filtration.

\subsubsection{2-Hydroxybenzaldehyde 2-[8-(2,3-diphenylpyrido[2,3-b]pyrazinyl)]hydrazone (2r)}

General Procedure 8 using 2-hydroxybenzaldehyde $(67 \mathrm{mg})$ gave $2 \mathbf{r}\left(\mathrm{R}_{\mathrm{f}}\left(\mathrm{CH}_{2} \mathrm{Cl}_{2}\right.\right.$-EtOAc 80:20) $\left.=0.44\right)$ in $60 \%$ yield as a yellow powder. Mp $>260^{\circ} \mathrm{C}$. IR: 952, 1019, 1096, 1163, 1233, 1270, 1309, 1328, 1422, 1540, 1562, 1594, 1618, 3064, $3317 \mathrm{~cm}^{-1} .{ }^{1} \mathrm{H}-\mathrm{NMR}\left(\mathrm{CDCl}_{3}\right): 6.96(\mathrm{td}, 1 \mathrm{H}, J=7.5$ and $1.1 \mathrm{~Hz}), 7.07(\mathrm{~d}, 1 \mathrm{H}$, $J=8.2 \mathrm{~Hz}), 7.23-7.44(\mathrm{~m}, 9 \mathrm{H}), 7.51-7.54(\mathrm{~m}, 2 \mathrm{H}), 7.58-7.62(\mathrm{~m}, 2 \mathrm{H}), 8.26(\mathrm{~s}, 1 \mathrm{H}), 8.91(\mathrm{~d}, 1 \mathrm{H}, J=5.3 \mathrm{~Hz})$, $9.71(\mathrm{br} \mathrm{s}, 1 \mathrm{H}), 10.60(\mathrm{br} \mathrm{s}, 1 \mathrm{H})$. The ${ }^{13} \mathrm{C}$ spectra could not be recorded due to low solubility in $\mathrm{CDCl}_{3}$ and DMSO. Anal. Calc. for $\mathrm{C}_{26} \mathrm{H}_{19} \mathrm{~N}_{5} \mathrm{O}$ (417.47): C 74.80, H 4.59, N, 16.78. Found: C 74.72, $\mathrm{H} 4.39$, $\mathrm{N}, 16.67$.

\subsubsection{Piperonal 2-[8-(2,3-diphenylpyrido[2,3-b]pyrazinyl)]hydrazone (2s)}

General Procedure 8 using piperonal $(83 \mathrm{mg})$ gave $2 \mathrm{~s}\left(\mathrm{R}_{\mathrm{f}}\left(\mathrm{CH}_{2} \mathrm{Cl}_{2}\right.\right.$-EtOAc 80:20) $\left.=0.37\right)$ in $70 \%$ yield as a yellow powder. Mp: $254^{\circ} \mathrm{C}$. IR: $933,1038,1150,1255,1339,1450,1489,1501,1545,1568,1590$, 2901, 3060, 3322, $3648 \mathrm{~cm}^{-1}$. ${ }^{1} \mathrm{H}-\mathrm{NMR}\left(\mathrm{CDCl}_{3}\right): 6.03(\mathrm{~s}, 2 \mathrm{H}), 6.84(\mathrm{~d}, 1 \mathrm{H}, J=8.0 \mathrm{~Hz}), 7.07(\mathrm{dd}, 1 \mathrm{H}, J=8.1$ and 1.6 Hz), 7.28-7.42 (m, 7H), $7.50(\mathrm{t}, 3 \mathrm{H}, J=6.6 \mathrm{~Hz}), 7.59(\mathrm{~d}, 2 \mathrm{H}, J=6.8 \mathrm{~Hz}), 7.97(\mathrm{~s}, 1 \mathrm{H}), 8.85(\mathrm{~d}, 1 \mathrm{H}$, $J=5.3 \mathrm{~Hz}), 9.66(\mathrm{~s}, 1 \mathrm{H}) .{ }^{13} \mathrm{C}-\mathrm{NMR}\left(\left(\mathrm{CD}_{3}\right)_{2} \mathrm{SO}\right): 101.5\left(\mathrm{CH}_{2}\right), 103.5(\mathrm{CH}), 104.9(\mathrm{CH}), 108.5(\mathrm{CH}), 123.0$ $(\mathrm{CH}), 125.6(\mathrm{C}), 128.1(2 \mathrm{CH}), 128.2(2 \mathrm{CH}), 128.8(\mathrm{CH}), 129.1(\mathrm{CH}), 129.2(\mathrm{C}), 129.7(2 \mathrm{CH}), 130.1(2 \mathrm{CH})$, 
$138.3(\mathrm{C}), 138.6(\mathrm{C}), 145.1(\mathrm{CH}), 147.7(\mathrm{C}), 148.1(\mathrm{C}), 148.8(\mathrm{C}), 149.6$ (C), $150.0(\mathrm{C}), 154.5(\mathrm{CH}), 155.5(\mathrm{C})$. Anal. Calc. for $\mathrm{C}_{27} \mathrm{H}_{19} \mathrm{~N}_{5} \mathrm{O}_{2}$ (445.48): C 72.80, H 4.30, N, 15.72. Found: C 72.95, H 4.44, N, 15.83.

3.12.4. 2-Hydroxy-4-methoxybenzaldehyde 2-[8-(2,3-diphenylpyrido[2,3-b]pyrazinyl)]hydrazone (2t)

General Procedure 8 using 2-hydroxy-4-methoxybenzaldehyde $(84 \mathrm{mg})$ gave $2 \mathbf{t}\left(\mathrm{R}_{\mathrm{f}}\left(\mathrm{CH}_{2} \mathrm{Cl}_{2}\right.\right.$ EtOAc 80:20) $=0.58$ ) in $80 \%$ yield as a yellow powder. $\mathrm{Mp}>260^{\circ} \mathrm{C}$. IR: 1032, 1135, 1163, 1238, 1291, 1339, 1431, 1439, 1461, 1510, 1543, 1566, 1631, 2845, 2931, 3004, 3056, 3176, $3317 \mathrm{~cm}^{-1} .{ }^{1} \mathrm{H}-\mathrm{NMR}\left(\mathrm{CDCl}_{3}\right)$ : $3.85(\mathrm{~s}, 3 \mathrm{H}), 6.52(\mathrm{dd}, 1 \mathrm{H}, J=8.5$ and $2.5 \mathrm{~Hz}), 6.58(\mathrm{~d}, 1 \mathrm{H}, J=2.5 \mathrm{~Hz}), 7.15(\mathrm{~d}, 1 \mathrm{H}, J=8.6 \mathrm{~Hz}), 7.19(\mathrm{~d}, 1 \mathrm{H}$, $J=5.2 \mathrm{~Hz}), 7.28-7.43(\mathrm{~m}, 6 \mathrm{H}), 7.50-7.54(\mathrm{~m}, 2 \mathrm{H}), 7.58-7.61(\mathrm{~m}, 2 \mathrm{H}), 8.19(\mathrm{~s}, 1 \mathrm{H}), 8.88(\mathrm{br} \mathrm{s}, 1 \mathrm{H}), 9.59$ (br s, $1 \mathrm{H}), 10.81(\mathrm{~s}, 1 \mathrm{H})$. The ${ }^{13} \mathrm{C}$ spectra could not be recorded due to low solubility in $\mathrm{CDCl}_{3}$ and DMSO. Anal. Calc. for $\mathrm{C}_{27} \mathrm{H}_{21} \mathrm{~N}_{5} \mathrm{O}_{2}$ (447.50): C 72.47, H 4.73, N, 15.65. Found: C 72.53, H 4.89, N, 15.60.

3.12.5. 4-(Trifluoromethyl)benzaldehyde 2-[8-(2,3-diphenylpyrido[2,3-b]pyrazinyl)]hydrazone (2u)

General Procedure 8 using 4-(trifluoromethyl)benzaldehyde $(87 \mathrm{mg})$ gave $2 \mathbf{u}\left(\mathrm{R}_{\mathrm{f}}\left(\mathrm{CH}_{2} \mathrm{Cl}_{2}\right.\right.$ - EtOAc $80: 20)=0.51)$ in $73 \%$ yield as a yellow powder. Mp: $258-260^{\circ} \mathrm{C}$. IR: 1017, 1066, 1109, 1124, 1145, 1236, 1300, 1321, 1512, 1545, 1562, 1588, 3060, 3184, 3317, $3652 \mathrm{~cm}^{-1} .{ }^{1} \mathrm{H}-\mathrm{NMR}\left(\mathrm{CDCl}_{3}\right): 7.28-7.43(\mathrm{~m}, 6 \mathrm{H})$, 7.50-7.53 (m, 2H), 7.56-7.61 (m, 3H), $7.68(\mathrm{~d}, 2 \mathrm{H}, J=8.2 \mathrm{~Hz}), 7.87(\mathrm{~d}, 2 \mathrm{H}, J=7.8 \mathrm{~Hz}), 8.11(\mathrm{~s}, 1 \mathrm{H})$, $8.91(\mathrm{~d}, 1 \mathrm{H}, J=5.2 \mathrm{~Hz}), 9.90$ (br s, $1 \mathrm{H}) .{ }^{13} \mathrm{C}-\mathrm{NMR}\left(\left(\mathrm{CD}_{3}\right)_{2} \mathrm{SO}, 333 \mathrm{~K}\right): 103.8(\mathrm{CH}), 124.0\left(\mathrm{q}, \mathrm{CF}_{3}\right.$, $J=272 \mathrm{~Hz}), 125.4(\mathrm{C}), 125.5(\mathrm{q}, 2 \mathrm{CH}, J=3.7 \mathrm{~Hz}), 127.1(2 \mathrm{CH}), 127.8(2 \mathrm{CH}), 127.9(2 \mathrm{CH}), 128.7(\mathrm{CH})$, $128.8(\mathrm{CH}), 129.2\left(\mathrm{q}, \mathrm{C}^{-\mathrm{CF}_{3}}, J=31.7 \mathrm{~Hz}\right), 129.5(2 \mathrm{CH}), 129.8(2 \mathrm{CH}), 138.1(\mathrm{C}), 138.4(\mathrm{C}), 138.5(\mathrm{C})$, $143.2(\mathrm{CH}), 147.4(\mathrm{C}), 149.4(\mathrm{C}), 150.3(\mathrm{C}), 154.3(\mathrm{CH}), 155.5(\mathrm{C})$. Anal. Calc. for $\mathrm{C}_{27} \mathrm{H}_{18} \mathrm{~F}_{3} \mathrm{~N}_{5}$ (469.47): C 69.08, H 3.86, N, 14.92. Found: C 69.25, H 3.97, N, 14.78.

\subsection{Nucleophilic Substitution Using a Phenolate: Methyl 2-[8-(2,3-diphenylpyrido[2,3-b]pyrazinyl)]oxy- 5-methoxybenzoate ( $2 v)$}

A mixture of 8-iodo-2,3-diphenylpyrido[2,3-b]pyrazine (2b-I, $0.20 \mathrm{~g}, 0.50 \mathrm{mmol}$ ), methyl 2-hydroxy-5-methoxy-benzoate $(0.10 \mathrm{~g}, 0.55 \mathrm{mmol}), \mathrm{K}_{2} \mathrm{CO}_{3}(77 \mathrm{mg}, 0.55 \mathrm{mmol})$ and DMSO (1 mL) was heated at $110{ }^{\circ} \mathrm{C}$ for $2 \mathrm{~h}$. The cooled residue was treated by an aqueous solution of $\mathrm{Na}_{2} \mathrm{CO}_{3}$ $(10 \mathrm{~mL})$ before extraction with $\mathrm{Et}_{2} \mathrm{O}(3 \times 10 \mathrm{~mL})$. The organic layer was dried over $\mathrm{MgSO}_{4}$, filtered and concentrated under reduced pressure, and the residue was chromatographed over silica gel (eluent: $\mathrm{CH}_{2} \mathrm{Cl}_{2}-\mathrm{MeOH}$ 95:5; $\mathrm{Rf}\left(\mathrm{CH}_{2} \mathrm{Cl}_{2}\right.$-EtOAc 95:5) $\left.=0.50\right)$ to give the title compound $2 \mathbf{v}$ in $64 \%$ yield as a beige powder. Mp: $206^{\circ} \mathrm{C}$. IR: 542, 698, 773, 856, 1021, 1072, 1109, 1205, 1235, 1263, 1333, 1350, 1434, 1468, 1496, 1554, 1594, 1719, 2845, 2956, $3041 \mathrm{~cm}^{-1} .{ }^{1} \mathrm{H}-\mathrm{NMR}\left(\mathrm{CDCl}_{3}\right): 3.65(\mathrm{~s}, 3 \mathrm{H}), 3.90(\mathrm{~s}, 3 \mathrm{H}), 6.64(\mathrm{~d}$, $J=5.2 \mathrm{~Hz}, 1 \mathrm{H}), 7.19(\mathrm{dd}, 1 \mathrm{H}, J=8.9,3.0 \mathrm{~Hz}), 7.24(\mathrm{~d}, 1 \mathrm{H}, J=9.5 \mathrm{~Hz}), 7.30-7.40(\mathrm{~m}, 6 \mathrm{H}), 7.55-7.58$ $(\mathrm{m}, 3 \mathrm{H}), 7.61-7.64(\mathrm{~m}, 2 \mathrm{H}), 8.86(\mathrm{~d}, 1 \mathrm{H}, J=5.2 \mathrm{~Hz}) .{ }^{13} \mathrm{C}-\mathrm{NMR}\left(\mathrm{CDCl}_{3}\right): 52.4\left(\mathrm{CH}_{3}\right), 56.0\left(\mathrm{CH}_{3}\right), 107.6$ $(\mathrm{CH}), 116.3(\mathrm{CH}), 120.6(\mathrm{CH}), 124.6(\mathrm{C}), 125.0(\mathrm{CH}), 128.2(2 \mathrm{CH}), 128.4(2 \mathrm{CH}), 129.0(\mathrm{C}), 129.1(\mathrm{CH})$, $129.4(\mathrm{CH}), 130.2(2 \mathrm{CH}), 130.3(2 \mathrm{CH}), 138.2(\mathrm{C}), 138.7(\mathrm{C}), 147.0(\mathrm{C}), 151.1(\mathrm{C}), 153.6(\mathrm{C}), 154.4(\mathrm{CH})$, 156.6 (C), 157.4 (C), 163.0 (C), 164.7 (C). Anal. Calc. for $\mathrm{C}_{28} \mathrm{H}_{21} \mathrm{~N}_{3} \mathrm{O}_{4}$ (463.49): C 72.56, H 4.57, N, 9.07 . Found: C 72.49, H 4.65, N, 9.01 .

\section{Conclusions}

Original pyrazino-fused polycyclic scaffolds were synthesized by combining deprotometalation-iodolysis with palladium- or copper-catalyzed couplings or direct substitution reactions. This study highlights the interest in preparing iodo derivatives of sensitive aromatic heterocycles by using lithium-zinc basic combinations to access scaffolds of potential biological interest. Interestingly, bromine and trichloroisocyanuric acid were successfully employed as electrophiles to intercept the intermediate heteroarylzinc halides.

Supplementary Materials: Supplementary materials are available online. 
Author Contributions: F.L., T.L., M.B., C.C., I.C., C.G. and J.L. synthesized and analyzed all compounds presented in this article; E.C. contributed to the identification of some synthesized compounds by NMR; E.L. and L.P. contributed with the experiments performed under microwave irradiation; L.P., M.S., B.B. and S.B. performed the bioassays; T.R. collected the X-ray diffraction data and solved the structures. F.M. wrote the paper with the help of E.C., L.P., V.T., S.R., S.B. and T.R.; F.L. started the project, designed the molecules and revised the paper.

Funding: This research received no external funding.

Acknowledgments: O. Lozach and T. Robert (KISSf screening facility) are acknowledged for their excellent technical support, and William Erb for his relevant advice. F.M. and F.L. thank the Université de Rennes 1. L.P. and V.T. thank the French Cancer League (Comité 17) for financial support and the Cancéropôle Grand Ouest (axis: natural sea products in cancer treatment) for scientific support. S.B. and S.R. thank the Cancéropôle Grand Ouest (axis: natural sea products in cancer treatment), IBiSA (French Infrastructures en sciences du vivant: biologie, santé et agronomie) and Biogenouest (Western France life science and environment core facility network) for supporting KISSf screening facility. We acknowledge FEDER founds (D8 VENTURE Bruker AXS diffractometer) and Thermofisher (generous gift of 2,2,6,6-tetramethylpiperidine).

Conflicts of Interest: The authors declare no conflict of interest.

\section{References}

1. Ajani, O.O. Present status of quinoxaline motifs: Excellent pathfinders in therapeutic medicine. Eur. J. Med. Chem. 2014, 85, 688-715. [CrossRef] [PubMed]

2. Antoine, M.; Schuster, T.; Seipelt, I.; Aicher, B.; Teifel, M.; Gunther, E.; Gerlach, M.; Marchand, P. Efficient synthesis of novel disubstituted pyrido[3,4-b]pyrazines for the design of protein kinase inhibitors. MedChem Comm 2016, 7, 224-229. [CrossRef]

3. Sonawane, N.D.; Rangnekar, D.W. Synthesis and application of 2-styryl-6,7-dichlorothiazolo[4,5- $b$ ] quinoxaline based fluorescent dyes: Part 3. J. Heterocycl. Chem. 2002, 39, 303-308. [CrossRef]

4. Thomas, K.R.J.; Velusamy, M.; Lin, J.T.; Chuen, C.-H.; Tao, Y.-T. Chromophore-labeled quinoxaline derivatives as efficient electroluminescent materials. Chem. Mater. 2005, 17, 1860-1866. [CrossRef]

5. Dailey, S.; Feast, W.J.; Peace, R.J.; Sage, I.C.; Till, S.; Wood, E.L. Synthesis and device characterisation of side-chain polymer electron transport materials for organic semiconductor applications. J. Mater. Chem. 2001, 11, 2238-2243. [CrossRef]

6. Lassagne, F.; Chevallier, F.; Mongin, F. Saccharin as an organocatalyst for quinoxalines and pyrido[2,3- $b]$ pyrazines syntheses. Synth. Commun. 2014, 44, 141-149. [CrossRef]

7. Gschwend, H.W.; Rodriguez, H.R. Heteroatom-facilitated lithiations. Org. React. 1979, 26, 1-360. [CrossRef]

8. Gant, T.G.; Meyers, A.I. The chemistry of 2-oxazolines (1985-present). Tetrahedron 1994, 50, $2297-2360$. [CrossRef]

9. Schlosser, M. Organometallics in Synthesis: A Manual; Schlosser, M., Ed.; John Wiley \& Sons: New York, NY, USA, 1994.

10. Hartung, C.G.; Snieckus, V. The directed ortho metalation reaction-A point of departure for new synthetic aromatic chemistry. Mod. Arene Chem. 2002, 330-367. [CrossRef]

11. Clayden, J. Directed metalation of aromatic compounds. Chem. Organolithium Compd. 2004, 1, 495-646. [CrossRef]

12. Tilly, D.; Magolan, J.; Mortier, J. Directed remote aromatic metalations: Mechanisms and driving forces. Chem. Eur. J. 2012, 18, 3804-3820. [CrossRef] [PubMed]

13. Queguiner, G.; Marsais, F.; Snieckus, V.; Epsztajn, J. Directed metalation of pi-deficient azaaromatics: Strategies of functionalization of pyridines, quinolines, and diazines. Adv. Heterocycl. Chem. 1991, 52, 187-304. [CrossRef]

14. Mongin, F.; Queguiner, G. Advances in the directed metallation of azines and diazines (pyridines, pyrimidines, pyrazines, pyridazines, quinolines, benzodiazines and carbolines). Part 1: Metallation of pyridines, quinolines and carbolines. Tetrahedron 2001, 57, 4059-4090. [CrossRef]

15. Schlosser, M.; Mongin, F. Pyridine elaboration through organometallic intermediates: Regiochemical control and completeness. Chem. Soc. Rev. 2007, 36, 1161-1172. [CrossRef] [PubMed]

16. Mokhtari Brikci-Nigassa, N.; Bentabed-Ababsa, G.; Erb, W.; Mongin, F. In situ "trans-metal trapping": An efficient way to extend the scope of aromatic deprotometalation. Synthesis 2018, 50, 3615-3633. [CrossRef]

17. Uzelac, M.; Mulvey, R.E. Trans-metal-trapping: Concealed crossover complexes en route to transmetallation? Chem. Eur. J. 2018, 24, 7786-7793. [CrossRef] [PubMed] 
18. L'Helgoual'ch, J.M.; Seggio, A.; Chevallier, F.; Yonehara, M.; Jeanneau, E.; Uchiyama, M.; Mongin, F. Deprotonative metalation of five-membered aromatic heterocycles using mixed lithium-zinc species. J. Org. Chem. 2008, 73, 177-183. [CrossRef] [PubMed]

19. García-Álvarez, P.; Mulvey, R.E.; Parkinson, J.A. "LiZn(TMP) $)_{3}$ ”, a zincate or a turbo-lithium amide reagent? DOSY NMR spectroscopic evidence. Angew. Chem. Int. Ed. 2011, 50, 9668-9671. [CrossRef] [PubMed]

20. Bisballe, N.; Hedidi, M.; Demmer, C.S.; Chevallier, F.; Roisnel, T.; Dorcet, V.; Halauko, Y.S.; Ivashkevich, O.A.; Matulis, V.E.; Bentabed-Ababsa, G.; et al. Functionalization of oxazolo[4,5-b] pyrazines by deprotometallation. Eur. J. Org. Chem. 2018, 3904-3913. [CrossRef]

21. Akimoto, G.; Otsuka, M.; Takita, R.; Uchiyama, M.; Hedidi, M.; Bentabed-Ababsa, G.; Lassagne, F.; Erb, W.; Mongin, F. Deprotonative metalation of methoxy-substituted arenes using lithium 2,2,6,6-tetramethylpiperidide: Experimental and computational study. J. Org. Chem. 2018. [CrossRef] [PubMed]

22. Amara, R.; Bentabed-Ababsa, G.; Hedidi, M.; Khoury, J.; Awad, H.; Nassar, E.; Roisnel, T.; Dorcet, V.; Chevallier, F.; Fajloun, Z.; et al. Synthesis of $N$-aryl and $N$-heteroaryl $\gamma_{-}^{-}, \delta$-, and $\varepsilon$-lactams using deprotometalation-iodination and $N$-arylation, and properties thereof. Synthesis 2017, 49, 4500-4516. [CrossRef]

23. Hedidi, M.; Erb, W.; Lassagne, F.; Halauko, Y.S.; Ivashkevich, O.A.; Matulis, V.E.; Roisnel, T.; Bentabed-Ababsa, G.; Mongin, F. Functionalization of pyridyl ketones using deprotolithiation-in situ zincation. RSC Adv. 2016, 6, 63185-63189. [CrossRef]

24. Hedidi, M.; Maillard, J.; Erb, W.; Lassagne, F.; Halauko, Y.S.; Ivashkevich, O.A.; Matulis, V.E.; Roisnel, T.; Dorcet, V.; Hamze, M.; et al. Fused systems based on 2-aminopyrimidines: Synthesis combining deprotolithiation-in situ zincation with $\mathrm{N}$-arylation reactions and biological properties. Eur. J. Org. Chem. 2017, 5903-5915. [CrossRef]

25. Held, I.; Xu, S.; Zipse, H. Modular design of pyridine-based acyl-transfer catalysts. Synthesis 2007, 1185-1196. [CrossRef]

26. Antoine, M.; Czech, M.; Gerlach, M.; Gunther, E.; Schuster, T.; Marchand, P. Preparation of novel 2,3,8-trisubstituted pyrido[3,4-b]pyrazines and pyrido[2,3-b]pyrazines. Synthesis 2011, 794-806. [CrossRef]

27. Miyaura, N.; Suzuki, A. Palladium-catalyzed cross-coupling reactions of organoboron compounds. Chem. Rev. 1995, 95, 2457-2483. [CrossRef]

28. Kotha, S.; Lahiri, K.; Kashinath, D. Recent applications of the Suzuki-Miyaura cross-coupling reaction in organic synthesis. Tetrahedron 2002, 58, 9633-9695. [CrossRef]

29. Van Baelen, G.; Meyers, C.; Lemière, G.L.F.; Hostyn, S.; Dommisse, R.; Maes, L.; Augustyns, K.; Haemers, A.; Pieters, L.; Maes, B.U.W. Synthesis of 6-methyl-6H-indolo[3,2-c]isoquinoline and 6-methyl-6Hindolo[2,3-c]isoquinoline: Two new unnatural isoquinoline isomers of the cryptolepine series. Tetrahedron 2008, 64, 11802-11809. [CrossRef]

30. Sorokin, V.I. Copper(I) catalyzed N-arylation of azoles, the recent developments. Mini-Rev. Org. Chem. 2008, 5, 323-330. [CrossRef]

31. Monnier, F.; Taillefer, M. Catalytic C-C, C-N, and C-O Ullmann-type coupling reactions. Angew. Chem. Int. Ed. 2009, 48, 6954-6971. [CrossRef] [PubMed]

32. Surry, D.S.; Buchwald, S.L. Dialkylbiaryl phosphines in Pd-catalyzed amination: A user's guide. Chem. Sci. 2011, 2, 27-50. [CrossRef] [PubMed]

33. Lefèvre, G.; Franc, G.; Tlili, A.; Adamo, C.; Taillefer, M.; Ciofini, I.; Jutand, A. Contribution to the mechanism of copper-catalyzed C-N and C-O bond formation. Organometallics 2012, 31, 7694-7707. [CrossRef]

34. Beletskaya, I.P.; Cheprakov, A.V. The complementary competitors: Palladium and copper in C-N cross-coupling reactions. Organometallics 2012, 31, 7753-7808. [CrossRef]

35. Bariwal, J.; Van der Eycken, E. C-N bond forming cross-coupling reactions: An overview. Chem. Soc. Rev. 2013, 42, 9283-9303. [CrossRef] [PubMed]

36. Monnier, F.; Taillefer, M. Copper-catalyzed C(aryl)-N bond formation. Top. Organomet. Chem. 2013, 46, 173-204. [CrossRef]

37. Sambiagio, C.; Marsden, S.P.; Blacker, A.J.; McGowan, P.C. Copper catalysed Ullmann type chemistry: from mechanistic aspects to modern development. Chem. Soc. Rev. 2014, 43, 3525-3550. [CrossRef] [PubMed]

38. Amal Joseph, P.J.; Priyadarshini, S. Copper-mediated C-X functionalization of aryl halides. Org. Process Res. Dev. 2017, 21, 1889-1924. [CrossRef] 
39. Buden, M.E.; Vaillard, V.A.; Martin, S.E.; Rossi, R.A. Synthesis of carbazoles by intramolecular arylation of diarylamide anions. J. Org. Chem. 2009, 74, 4490-4498. [CrossRef] [PubMed]

40. Van Baelen, G.; Hostyn, S.; Dhooghe, L.; Tapolcsányi, P.; Mátyus, P.; Lemière, G.; Dommisse, R.; Kaiser, M.; Brun, R.; Cos, P.; et al. Structure-activity relationship of antiparasitic and cytotoxic indoloquinoline alkaloids, and their tricyclic and bicyclic analogues. Bioorg. Med. Chem. 2009, 17, 7209-7217. [CrossRef] [PubMed]

41. Teo, Y.-C.; Yong, F.-F.; Sim, S. Ligand-free $\mathrm{Cu}_{2} \mathrm{O}$-catalyzed cross coupling of nitrogen heterocycles with iodopyridines. Tetrahedron 2013, 69, 7279-7284. [CrossRef]

42. Hedidi, M.; Erb, W.; Bentabed-Ababsa, G.; Chevallier, F.; Picot, L.; Thiéry, V.; Bach, S.; Ruchaud, S.; Roisnel, T.; Dorcet, V.; et al. Synthesis of $N$-pyridyl azoles using a deprotometalation-iodolysis- $N$-arylation sequence and evaluation of their antiproliferative activity in melanoma cells. Tetrahedron 2016, 72, 6467-6476. [CrossRef]

43. Aukunuru, J.; Eedula, K.; Pasham, V.; Katla, V.; Reddy, S.K. Synthesis of novel piperonal derivatives and evaluation of their anticonvulsant activity using a nanoparticular formulation. Int. J. Pharm. Sci. Nanotechnol. 2009, 2, 435-442.

44. Mokhtari Brikci-Nigassa, N.; Bentabed-Ababsa, G.; Erb, W.; Chevallier, F.; Picot, L.; Vitek, L.; Fleury, A.; Thiery, V.; Souab, M.; Robert, T.; et al. 2-Aminophenones, a common precursor to $N$-aryl isatins and acridines endowed with bioactivities. Tetrahedron 2018, 74, 1785-1801. [CrossRef]

45. Klaeger, S.; Heinzlmeir, S.; Wilhelm, M.; Polzer, H.; Vick, B.; Koenig, P.-A.; Reinecke, M.; Ruprecht, B.; Petzoldt, S.; Meng, C.; et al. The target landscape of clinical kinase drugs. Science 2017, 358, 1148. [CrossRef] [PubMed]

46. Gottlieb, H.E.; Kotlyar, V.; Nudelman, A. NMR chemical shifts of common laboratory solvents as trace impurities. J. Org. Chem. 1997, 62, 7512-7515. [CrossRef] [PubMed]

47. Altomare, A.; Burla, M.C.; Camalli, M.; Cascarano, G.L.; Giacovazzo, C.; Guagliardi, A.; Moliterni, A.G.G.; Polidori, G.; Spagna, R. SIR97: A new tool for crystal structure determination and refinement. J. Appl. Crystallogr. 1999, 32, 115-119. [CrossRef]

48. Sheldrick, G.M. SHELXT-Integrated space-group and crystal-structure determination. Acta Crystallogr. Sect. A 2015, 71, 3-8. [CrossRef] [PubMed]

49. Sheldrick, G.M. Crystal structure refinement with SHELXL. Acta Crystallogr. Sect. C 2015, 71, 3-8. [CrossRef] [PubMed]

50. Van der Sluis, P.; Spek, A.L. BYPASS: An effective method for the refinement of crystal structures containing disordered solvent regions. Acta Crystallogr. Sect. A 1990, A46, 194-201. [CrossRef]

51. Spek, A.L. Single-crystal structure validation with the program PLATON. J. Appl. Crystallogr. 2003, 36, 7-13. [CrossRef]

52. Farrugia, L.J. ORTEP-3 for windows-A version of ORTEP-III with a graphical user interface (GUI). J. Appl. Crystallogr. 1997, 30, 565. [CrossRef]

53. Kjonaas, R.A.; Hoffer, R.K. Regiospecific 1,4-addition with Grignard-derived mixed triorganozincate reagents. J. Org. Chem. 1988, 53, 4133-4135. [CrossRef]

54. Zhang, X.-Z.; Wang, J.-X.; Bai, L. Microwave-assisted synthesis of quinoxalines in PEG-400. Synth. Commun. 2011, 41, 2053-2063. [CrossRef]

Sample Availability: Samples of the synthesized compounds are available from the corresponding authors.

(C) 2018 by the authors. Licensee MDPI, Basel, Switzerland. This article is an open access article distributed under the terms and conditions of the Creative Commons Attribution (CC BY) license (http:// creativecommons.org/licenses/by/4.0/). 